\title{
NETISCE: A Network-Based Tool for Cell Fate Reprogramming
}

\author{
Lauren Marazzi ${ }^{1}$ \\ Milan Shah ${ }^{1}$ \\ Shreedula Balakrishnan ${ }^{1}$ \\ Ananya Patil ${ }^{1}$ \\ Paola Vera-Licona ${ }^{1,2,3,4,{ }^{*}}$
}

${ }^{1}$ Center for Quantitative Medicine, ${ }^{2}$ Department of Cell Biology, ${ }^{3}$ Center for Cell Analysis and Modeling, and ${ }^{4}$ Institute for Systems Genomics, University of Connecticut School of Medicine, Farmington, CT 06030, USA

* corresponding author, veralicona@uchc.edu 


\section{ABSTRACT}

The search for effective therapeutic targets in fields like regenerative medicine and cancer research has generated interest in cell fate reprogramming. This cellular reprogramming paradigm can drive cells to a desired target state from any initial state. However, methods for identifying reprogramming targets remain limited for biological systems that lack large sets of experimental data or a dynamical characterization. We present NETISCE, a novel computational tool for identifying cell fate reprogramming targets in static networks. NETISCE identifies reprogramming targets through the innovative use of control theory within a dynamical systems framework. Through validations in studies of cell fate reprogramming from developmental, stem cell, and cancer biology, we show that NETISCE can predict previously identified cell fate reprogramming targets and identify potentially novel combinations of targets. NETISCE extends cell fate reprogramming studies to larger-scale biological networks without the need for full model parameterization and can be implemented by experimental and computational biologists to identify parts of a biological system that are relevant for the desired reprogramming task. 


\section{INTRODUCTION}

2 Cell reprogramming redefines a cell's identity by altering its epigenetic or transcriptional

3 landscapes through the forced expression of transcription factors, small molecules, non-coding

4 RNAs, or microenvironment-mediated changes. One type of cellular reprogramming, cell fate

5 reprogramming, aims to control the internal state of a cell so that it is driven from a selected

6 state to a target state or phenotype ${ }^{1-7}$. Practical applications of cell reprogramming in stem cell

7 engineering ${ }^{8-10}$ and cancer biology ${ }^{11-13}$ have generated a great interest in the task of cell-fate

8 reprogramming. Identifying combinations of reprogramming targets is especially useful for

9 treating complex diseases, regenerating tissue, or reversing acquired resistance to canonical

10 treatment regimens, where multi-drug approaches may be more effective than single drug

11 therapy ${ }^{1,14-18}$.

12 Genome-wide and computational systems biology approaches are being broadly adopted for

13 cell fate reprogramming studies. The majority of methods can be divided between those that

14 take a network-based approach ${ }^{19-24}$ and those that take a dynamical systems-based

15 approach ${ }^{25-30}$ to the task of cell fate reprogramming ${ }^{30-37}$. However, these methods either do not

16 capture all the essential information for cellular reprogramming ${ }^{38}$, or require mechanistic details

17 and kinetic parameters to build a mathematical model of the system (for a brief overview of

18 these approaches, please see Supplementary Text 1 and Supplementary Figure 1). 


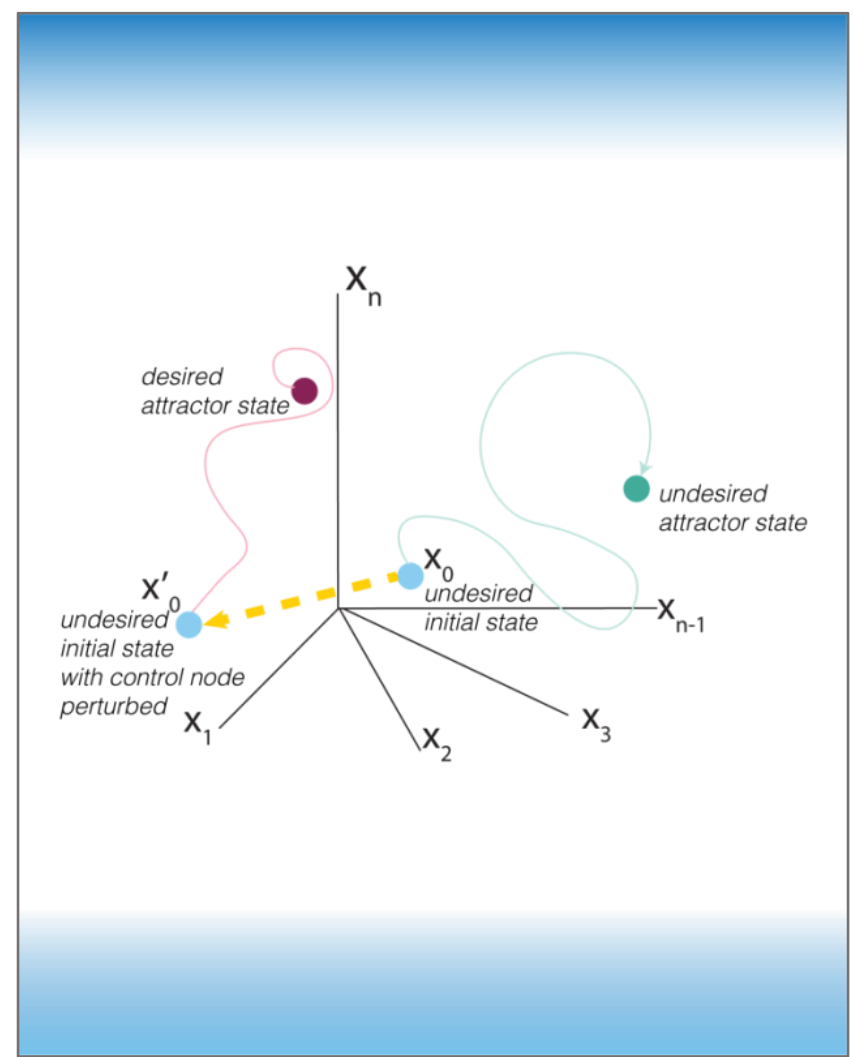

Fig.1 Control Theory View of Cell Fate Reprogramming. The Feedback Vertex Set control is a structure-based control method that can be applied to cell-fate reprogramming. By performing appropriate concerted perturbations to the minimal Feedback Vertex set on an initial state of the system that leads to an undesired attractor (green circle), the system can be shifted (yellow dashed lines) to a trajectory that leads to a desired attractor (purple circle).

19 Though many network-based and dynamical systems-based methods take a trial-and-error

20 approach to identify reprogramming targets, cell fate reprogramming can be viewed as a

21 classical attractor-based control theory problem. The goal here is to systematically determine

22 how to shift the cell's system from one attractor (stable state) to another with some degree of

23 optimality. Previous theoretical studies on the controllability of systems show that even for large,

24 non-linear biological systems, few targets need to be controlled to guide a system towards a

25 biologically admissible target state $e^{3,5}$. This has been shown experimentally in cell fate

26 reprogramming studies, such as in the transition of embryonic stem cells to somatic cells via

27 knockdown of pluripotency-associated transcription factors ${ }^{39}$, the reversion of tumorigenesis by

28 impairment of oncogenic signaling ${ }^{40}$, and fibroblast cell reprogramming ${ }^{41}$. The use of control

29 theory to identify cellular reprogramming targets has been limited and is not directly applicable

30 to large cell signaling networks ${ }^{42,43}$. Among the reasons for this limitation is the scarcity of 
31 available mechanistic details and kinetic parameters to build a mathematical model of the

32 system and, when the mechanistic rules are known, linear functions are used to describe them;

33 however, it is unclear how the commonly observed switch-like behavior of biochemical

34 processes $^{44,45}$, can influence the results ${ }^{1,27,46}$.

35 A new method for identifying attractor-based reprogramming targets using only network

36 topology extends from Control Theory for non-linear dynamics. The Feedback Vertex Set (FVS)

37 Control is a structure-based, attractor-based control method especially suited to non-linear

38 dynamical systems ${ }^{5}$. A network's minimal FVS is the minimal set of nodes that intersects all

39 cycles in a graph. FVS control states that appropriate perturbations on an FVS, which we refer

40 to herein as FVS control nodes, can drive the system from any arbitrary initial state to any of the

41 attractors of that system (Fig 1).

42 While the FVS control method provides a powerful cell fate reprogramming framework, it does

43 not identify the specific perturbations needed on FVS control nodes (knockouts or

44 overexpressions) to drive the system towards a specific set of attractors. By proceeding

45 analogously to Boolean networks, estimating the system's attractor landscape can aid in the

46 search for the perturbations needed to be applied on FVS control nodes. To that end, signal

47 flow estimation algorithms aim to estimate steady-states without full dynamical information of

48 the system. They can be useful to evaluate the effect of node perturbations in static

49 networks ${ }^{47,48}$. The Signal Flow Analysis (SFA) method is especially suited to estimate system

50 dynamics for non-linear complex systems ${ }^{47}$, and its application to biology has been recently

51 explored ${ }^{47,49}$. SFA successfully reproduced the steady-states of signaling networks derived from

52 ODE models and the changes in expression for network elements under different perturbations

53 from perturbation biology experiments with up to $80 \%$ accuracy $^{47}$. Latterly, SFA was applied to

54 an aging-related gene regulatory network (GRN) to identify potential aging reversion targets ${ }^{49}$.

55 Though Lee and colleagues did not take a control theory-based approach, aging reversion

56 targets were predicted by evaluating SFA-simulated single node perturbations that decreased 
57 the estimated steady-state expression values of aging-related biomarker nodes compared to an

58 unperturbed simulation.

59 Previously, we introduced OCSANA+ (Optimal Control and Simulation of Signaling Networks

60 from Network Analysis), a Cytoscape application that implements an FVS finding algorithm and

61 SFA to perform perturbation analyses on static networks ${ }^{50}$. OCSANA+ can be used to observe

62 the effect of perturbations on FVS control nodes for biological networks in a user-friendly

63 graphical interface. However, the application cannot customize the SFA algorithm to include

64 gene expression data. Additionally, the current software requires the user to configure every

65 perturbation simulation manually.

66 In this work, we introduce NETISCE (NETwork-drlven analysiS of CEllular reprogramming), a

67 novel computational method for identifying cell fate reprogramming targets. Our method can be

68 applied to any static network and only requires gene expression data from the undesired

69 phenotype for the initial state of the system. NETISCE employs the FVS control, Signal Flow

70 Analysis, and machine learning methods to identify FVS control nodes and their specific

71 combinations of perturbations that drive the system towards the desired cell fate.

72 To illustrate and validate our approach, we apply NETISCE to three very different examples of

73 cell fate reprogramming in the fields of developmental, stem cell, and cancer biology using both

74 GRNs and signaling networks. We show that NETISCE can reproduce the results of

75 experimentally validated cell fate reprogramming studies and identify new reprogramming

76 targets and perturbations.

77 We conclude that NETISCE extends the usefulness of static biological networks to analyses

78 that currently require full parameterization. This is implemented by approaching cell fate

79 reprogramming from the framework of control theory and dynamical systems, and applying

80 these concepts to a network-based analysis. Our method provides a useful and informative step

81 for researchers designing experimental or mathematical modeling studies of cell fate

82 reprogramming by identifying parts of the system relevant to the desired reprogramming task. 
83 NETISCE is a user-friendly tool implemented as a command-line Nextflow pipeline and Galaxy

84 Project workflow that non-experts can use in modeling or computational approaches to analyze

85 the biological systems of their interest.

\section{RESULTS}

87 NETISCE identifies combinations of perturbations to be applied on a GRN or signaling network

to trigger a shift from an undesired to the desired cell fate (Figure 2a). The core of the pipeline is

(1) the application of the FVS control to identify reprogramming targets and (2) an attractor

landscape estimation coupled with machine learning methods to predict the precise

91 perturbations that drive the system from an initial state (that would lead to an attractor

92 associated with an undesired phenotype) and towards an attractor associated with the desired

93 phenotype.

\section{Method's Validation}

\section{Reproducing experimentally validated perturbations to FVS control nodes for Cell Fate}

96 Specification in Ascidian Embryos. Using a GRN of cell fate specification in ascidian

97 embryos, Kobayashi and colleagues experimentally verified that concerted perturbations to the

98 network's FVS could induce embryonic cells to the epithelial, mesenchymal, endodermal,

99 notochord, brain, and pan-neural, and muscle tissue fates ${ }^{51,52}$. We performed simulations of the

100 experimentally verified perturbations on FVS control nodes for cell fate specification in ascidian

101 embryos using SFA.

102 The ascidian embryo GRN contained 92 nodes and 329 edges (Figure 3a). We identified 26

103 FVSes within the ascidian embryo GRN, including the set of six FVS control nodes

104 experimentally tested by Kobayashi et al. ${ }^{52}$ : Foxa.A, Foxd, Erk Signaling, Neurog, Tbx6-r.b, and

105 Zic-r.b. Without available normalized expression data, we simulated in silico unperturbed

106 embryonic development and the seven experimentally validated combinations of perturbations

107 (synchronous overexpression and knockout simulations) on FVS control nodes that induced the 


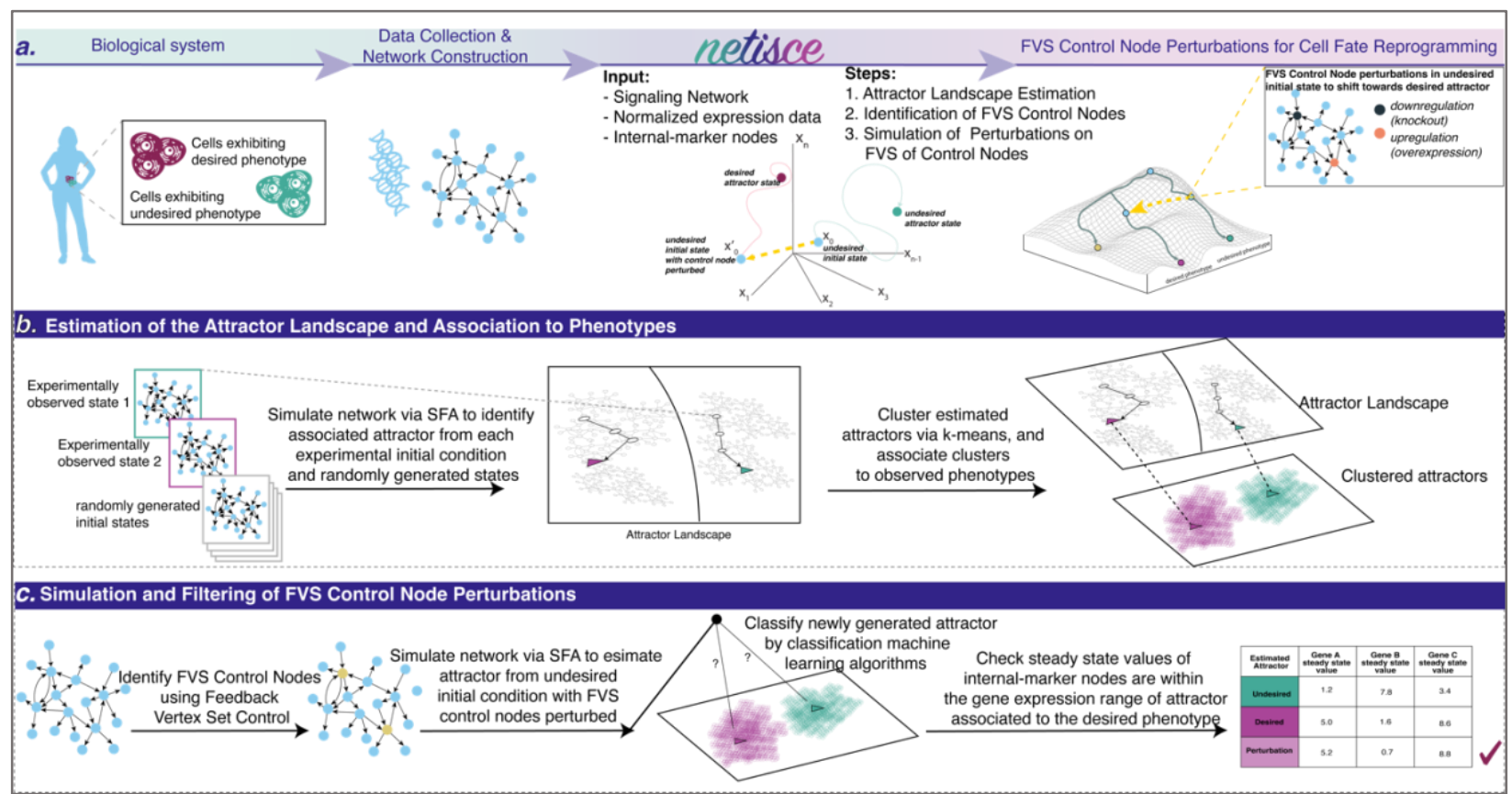

Fig. 2 NETISCE Pipeline and Method Overview. a Researchers can collect data from cells exhibiting desired and undesired phenotypes, and construct the signaling and regulatory networks governing cell reprogramming processes. The outputs of NETISCE are the combinations of network perturbations that shift the system from an undesired to a desired phenotype. b With the Signal Flow Analysis (SFA) algorithm ${ }^{47}$, in the first step, attractors are estimated by simulating the network with the initial states from normalized expression data and randomly generated initial states. The attractors are clustered via k-means and the clusters are associated with desired (purple) and undesired (green) phenotypes. $\mathbf{c}$ In the second step, FVS control nodes are identified using an FVS-finding algorithm ${ }^{53}$. Perturbations on FVS control nodes are performed by setting the initial states of the system to the gene expression value of the undesired phenotype, and overriding the states of FVS control nodes. In the third step, the sets of perturbations on FVS control nodes that achieve the desired reprogramming are identified using two filtering criteria. In the first criterion, the attractors generated from the perturbations on FVS control nodes are filtered using machine learning classification algorithms to obtain a set of perturbations whose attractors shifted from the cluster associated with the undesired phenotype to the cluster associated with the desired phenotype. In the second criterion, for user-defined internal-marker nodes, the steady-state expression values of the perturbations that passed the first filtering criterion (light purple) are evaluated to determine if their values have shifted to the gene expression range of the attractors associated to the desired phenotype.

embryonic tissue fates using SFA. To evaluate the perturbations, we analyzed the steady-state

expression values of the seven internal-marker nodes (one marker for each tissue) representing

genes measured in the experimental study (Figure 3b, Supplementary Table 1). Attractors

estimated by SFA can be compared analogously to the logarithm of the fold-change $\left(\log _{2} F C\right)$ in

112 differential gene expression analysis ${ }^{47}$, where the difference between the steady-state

113 expression value of a node in attractors generated from different initial states and/or

114 perturbations indicates that the gene expression of the node is upregulated if the difference is

115 positive, or downregulated if the difference is negative (see Methods for details). 


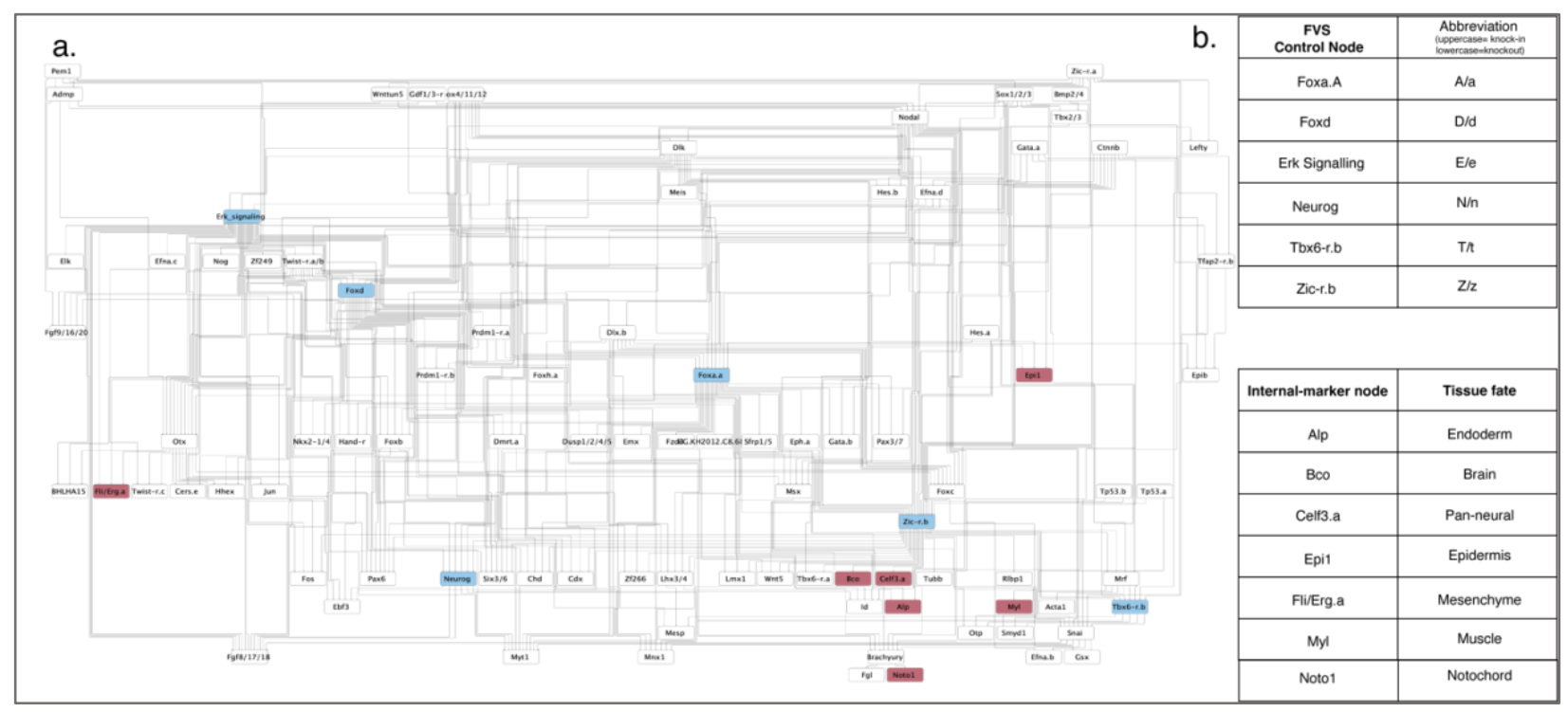

Fig. 3 Cell Fate Specification in Ascidian Embryo Model. a Gene regulatory network of cell fate specification in the Ascidian Embryo ciona intestinalis from Kobayashi et a. ${ }^{22}$. The network contains 92 nodes and 329 edges. Nodes highlighted in blue are FVS control nodes, and nodes colored in magenta are the internal-marker nodes used to evaluate if the perturbations on FVS control nodes successfully specified the desired cell fate. $\mathbf{b}$ The six control nodes identified by Kobayashi et al., which comprise the network's minimal Feedback Vertex Set (FVS). Uppercase or lowercase abbreviations for each node indicate up or downregulation of the FVS control node in a given combination of perturbations (see Figure 4). These nodes are used as the FVS control nodes in our simulations. c The seven internal-marker nodes and the respective tissue fates where they are upregulated as identified by Kobayashi et al. experimental studies. These nodes were also used to identify successful perturbations in our simulations.

117 We successfully reprogrammed the unperturbed embryo to six of the seven tissue fates using

118 the corresponding experimentally verified perturbations on FVS control nodes, giving the SFA in

119 our pipeline an overall 85\% accuracy (Figure 4, Supplementary Table 2). We could not

120 reproduce reprogramming to the pan-neural tissue fate. However, the internal-marker node for

121 the pan-neural cell fate (Celf3.a) was upregulated when we simulated the perturbation on FVS

122 control nodes that experimentally induced the brain+pan-neural tissue fates. 




Fig. 4 SFA simulations of perturbations on FVS control nodes for cell fate specification in Ascidian Embryos. Kobayashi et al. ${ }^{52}$ performed experimental combinations of perturbations on FVS control nodes to induce seven tissue fates in ascidian embryos. We aimed to reproduce these results in silico by simulating the combinations of perturbations on FVS control nodes using SFA. The results of each combination of perturbations are displayed in the radar plot. Each axis on the radar plot displays the steady-state expression value for an internal-marker node, with the tissue each internal-marker node represents in parentheses. The blue polygon is the steady-state expression values of the internalmarker nodes in the attractor associated with the unperturbed state. The yellow polygon displays the internal-marker node steady-state expression values produced by applying a combination of perturbations on FVS control nodes to the unperturbed initial state. The outer colored ring denotes the combination of perturbations on FVS control nodes performed, and which results are displayed at that axis. The tissue that was induced experimentally by a perturbation is denoted in parentheses. Each letter and capitalization stands for a separate FVS control node and its perturbed state, as described in the bottom table. For a simulation of the perturbations on FVS control nodes to be considered successful, the steady-state expression value of the internal-marker node must be greater in the attractor produced by the perturbation on the FVS control nodes than the steady-state expression value in the attractor associated with the unperturbed state (the yellow polygon extends out past the blue polygon on the radar plot). We were able to reproduce the cell fate specification results for $6 / 7$ cell fates, excluding adent $Z$ inducing the pan-neural cell fate.

\section{Identification of perturbations on FVS control nodes for induced pluripotent stem cell}

125 reprogramming from primed to naïve pluripotency. The mechanisms that maintain stem

126 cells' pluripotency or signal development are complex. Yachie-Kinoshita and colleagues 


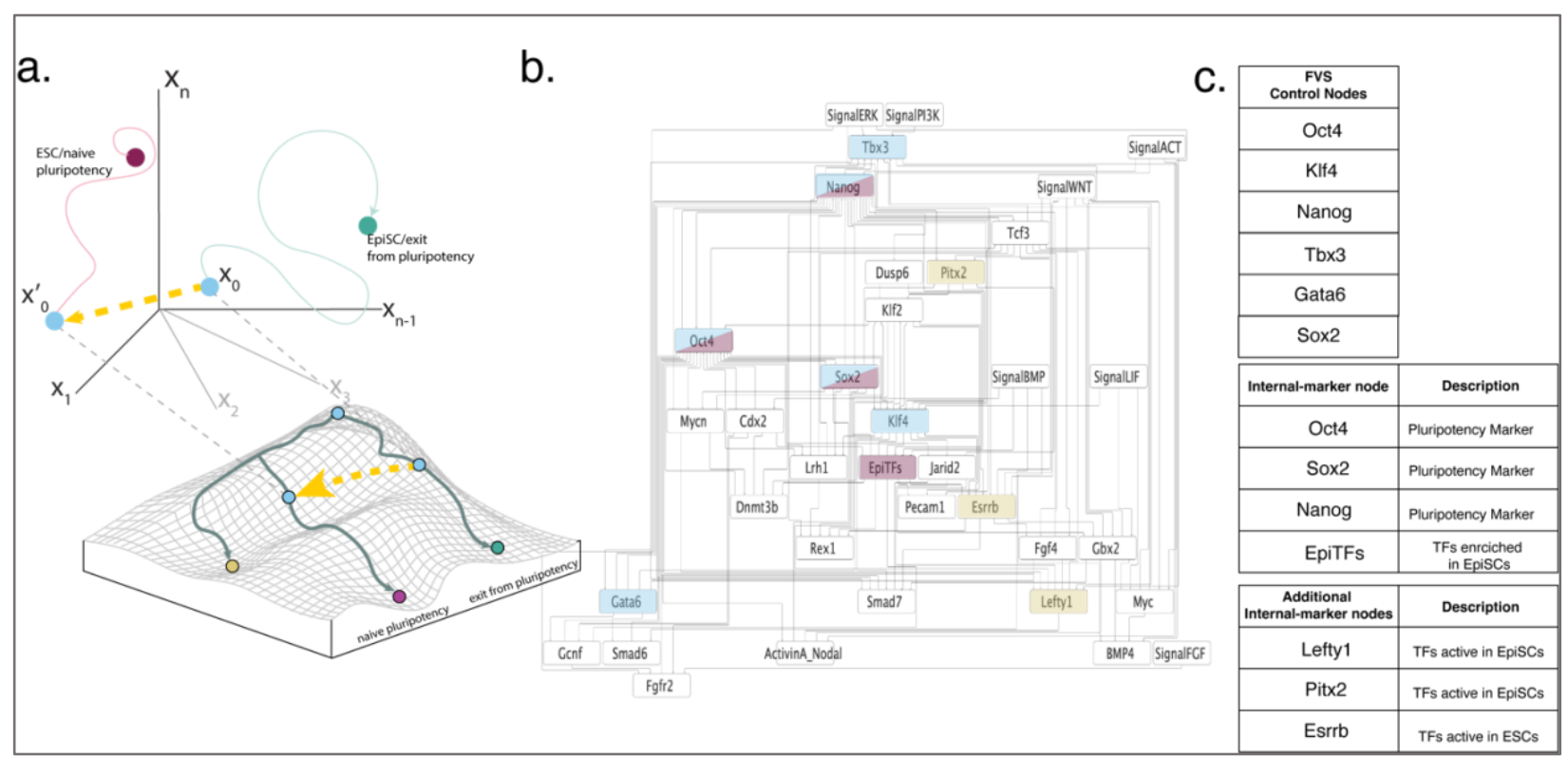

Fig 5. Reprogramming of Epiblast stem cells (EpiSCs) to Embryonic Stem Cells (ESCs). a The goal of this study is for NETISCE to identify the perturbations on FVS control nodes that shift EpiSCs towards ESCs, reverting from a primed to a naïve pluripotent state. To that end, we initialize the system with the gene expression data from EpiSC cells $\left(x_{0}\right)$, and, with the correct combination of perturbations on FVS control nodes, shift the system to a state (yellow arrow leading to $x_{0}^{\prime}$ ) that leads to the ESC cell fate (purple circle). b Pluripotent Stem Cell signaling network as constructed by Yachie-Kinoshita et al. ${ }^{54}$. The network contains 36 nodes and 143 edges. Nodes colored in blue are FVS control nodes, nodes with magenta coloring are the internal-marker nodes used by Yachie-Kinoshita et al., and gold nodes are the additional internal-marker nodes we used for further selection of perturbations on FVS control nodes. Note that the nodes Sox2, Nanog, and Oct4 are FVS control nodes and internal-marker nodes, and thus colored with both blue and purple. $\mathrm{c}$ Key nodes for the desired cell fate reprogramming. There are six FVS control nodes within the system. Four internal-marker nodes were used by Yachie-Kinoshita et al. to identify successful reprogramming targets for the ESC state. We identified an additional three internal-marker nodes to filter perturbations on FVS control nodes. For the internal-marker nodes, we denote which behavior they represent in pluripotency signaling in the second column.

134 The pluripotency signaling network contained 36 nodes and 143 edges (Figure 5b). Using

135 NETISCE, we estimated the attractors from EpiSC and ESC gene expression data and attractors from 100,000 randomly generated initial states. On these 100,006 attractors, we

137 performed k-means clustering. The optimal number of clusters identified by the elbow and 
silhouette metrics was $\mathrm{k}=2$. One cluster contained the attractors generated from the initial state values of the EpiSC cells gene expression. The second cluster included the attractors

140 generated from the ESC cell gene expression initialization.

141 NETISCE identified one FVS in the network, comprising six nodes: Nanog, Oct4, Klf4, Sox2,

142 Gata6, and Tbx3 (Figure 5c). Then, we simulated the 729 combinations of perturbations on the

143 FVS control nodes. Of the 729 perturbations, 375 passed the machine learning classification

144 filtering criterion. Here, we used the same four internal-marker nodes as Yachie-Kinoshita et al.:

145 Oct4, Sox2, and Nanog as markers of naïve pluripotency, and EpiTFs as the marker of enriched 146 transcription factors in EpiSCs. The steady-state expression values of the internal-marker nodes

147 in 132 of the 375 attractors calculated from the perturbations on FVS control nodes were in the 148 range of gene expression values of the ESC associated attractors and thus passed criterion 2

149 for all replicates. Notably, one perturbation on FVS control nodes that passed both filtering 150 criteria - overexpression of Nanog — was also identified and experimentally validated by

151 Yachie-Kinoshita et al. (Figure 6a). In the Boolean simulations and experimental validation by

152 Yachie-Kinoshita et al., Klf4 overexpression also induced the ESC fate. Although Klf4 was an

153 FVS control node in the network and its overexpression passed the machine learning filtering

154 criterion, the perturbation did not pass the internal-marker node filtering criterion (Figure 6a). In

155 this case, the steady-state expression values of Nanog, Sox2, and Oct4 (when considered as

156 internal-marker nodes) did not reach the gene expression levels of the ESC state (Figure 6a).

157 Overall, we show that overexpression of the FVS control node Nanog results in cell fate

158 reprogramming to naïve pluripotency, in agreement with the results from the Boolean Model

159 simulations and experimental validations.

160 We explored the ability to further filter the perturbations on FVS control nodes by increasing the

161 number of internal-marker nodes. We identified three additional nodes from gene expression

162 data provided by Yachie-Kinoshita et al. These included Lefty1, Pitx2 (transcription factors

163 active in EpiSCs), and Esrrb (a transcription factor active in ESCs). This reduced the 132 
bioRxiv preprint doi: https://doi.org/10.1101/2021.12.30.474582; this version posted January 1, 2022. The copyright holder for this preprint (which was not certified by peer review) is the author/funder, who has granted bioRxiv a license to display the preprint in perpetuity. It is made available under aCC-BY-NC 4.0 International license.

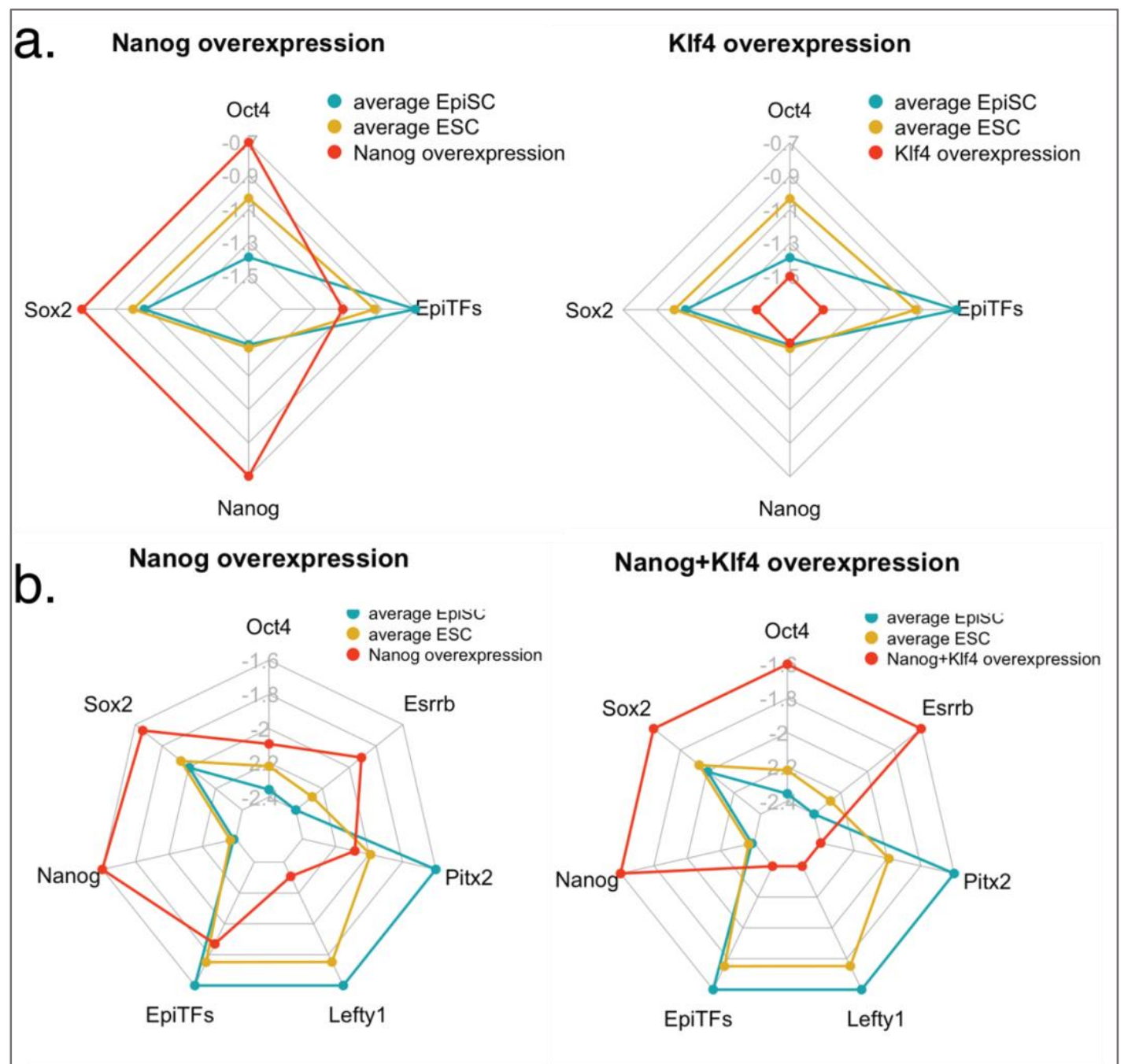

Fig. 6 Results of simulations of perturbations on FVS control nodes for EpiSC reprogramming to ESCs. Radar plots for SFA simulations of perturbations on FVS control nodes in the pluripotent stem cell model. The title of each plot indicates the perturbation on FVS control nodes. Each axis on the radar plot displays the steady-state expression value for an internal-marker node. The blue polygon are the average steady-state expression values of the internal-marker nodes for attractors generated from the EpiSC experimental samples. The yellow polygon are the average steady-state expression values of the internal-marker nodes for attractors generated from the ESC experimental samples. The orange polygon are the steady-state expression values of the internal-marker nodes in the attractor produced by the specified perturbation on FVS control nodes. A perturbation is considered successful if $90 \%$ of the internal-marker nodes have steady-state expression values within the gene expression range of the ESCassociated attractor. In other words, if the yellow polygon at the axis of a specific internal-marker node extends beyond the blue polygon, the orange polygon must extend beyond the yellow polygon at that internal-marker axis. Conversely, if the yellow polygon at the axis of a specific internal-marker node does not extend past the blue polygon, the orange polygon must not extend past the yellow polygon at that internal-marker axis. a Radar plots using the four internal-marker nodes used in Yachie-Kinoshita et al. for the perturbations of Nanog overexpression and Klf4 overexpression. Klf4 overexpression does not pass the internal-marker node filtering criteria because the steadystate expression values of Oct4, Sox2, and Nanog are not within the range of expression of these genes in the attractor produced from ESC gene expression data. b Radar plots using the four internal-marker nodes used in Yachie-Kinoshita et al., and the additional 3 internal-marker nodes identified from differential gene expression data for two successful perturbations - Nanog overexpression and combined Nanog+Klf4 overexpression. 
combination of Nanog+Klf4 overexpression were two of the fifteen perturbations that passed the

167 internal-marker node criteria (Figure 6b, Supplementary Table 3). The combination of

168 Nanog+Klf4 was not previously identified by Yachie-Kinoshita et al. However, combination Klf4

169 and Nanog overexpression may play an essential role in maintaining pluripotency, as Klf4 links

170 extracellular signaling information to positively regulate downstream Nanog transcription, and

171 overexpression of Nanog was found to rescue pluripotency in the case of Klf4 knockdown ${ }^{55}$.

172 Identification of perturbations on FVS control nodes to overcome adaptive resistance to

173 targeted MAPK inhibitor therapy in colorectal cancers. BRAF inhibition (BRAFi) therapy is a

174 form of MAPK inhibitor (MAPKi) therapy used to treat cancer patients with mutant BRAF. BRAFi

175 inhibits the MAPK signaling pathway, suppressing proliferation and inducing apoptosis. In

176 colorectal cancers (CRCs), adaptive resistance emerges against BRAFi through the activation

177 of MAPK signaling by upstream regulator EGFR. Park et al. were interested in identifying a

178 gene that, when perturbed in combination with BRAFi, could prevent the development of

179 adaptive resistance to BRAFi ${ }^{56}$. However, instead of inhibiting upstream molecules like in the

$180 \mathrm{BRAFi}+\mathrm{EGRFi}$ treatment, they searched for a target within the MAPK signaling pathway that

181 could sensitize HT29 CRC cells to MAPKi therapy. By constructing a Boolean model of

182 signaling pathways in CRC and simulating perturbations to every node in the model, Park et al.

183 showed that BRAFi combined with SRC inhibition (SRCi) prevented the development of

184 adaptive resistance via inhibition of ERK (MAPK1), a member of the MAPK signaling pathway.

185 This result was validated experimentally in HT29 CRC cells. Therefore, with only the CRC signal

186 pathway network structure, RNA-seq data from untreated HT29 cells, and functionally annotated

187 mutational information as input to NETISCE, we sought to identify perturbations on FVS control

188 nodes in combination with BRAFi that overcome adaptive resistance to MAPKi therapy (Figure

$1897 \mathrm{a})$.

190 Park and colleagues built a network of CRC signaling pathways containing 95 nodes and 337

191 edges (Figure $7 b$ ). We adapted the PROFILE method ${ }^{57}$ to verify that the generic CRC network 
a.

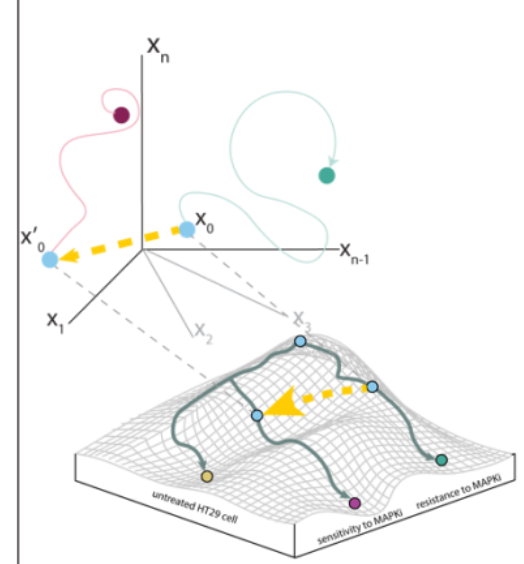

b.

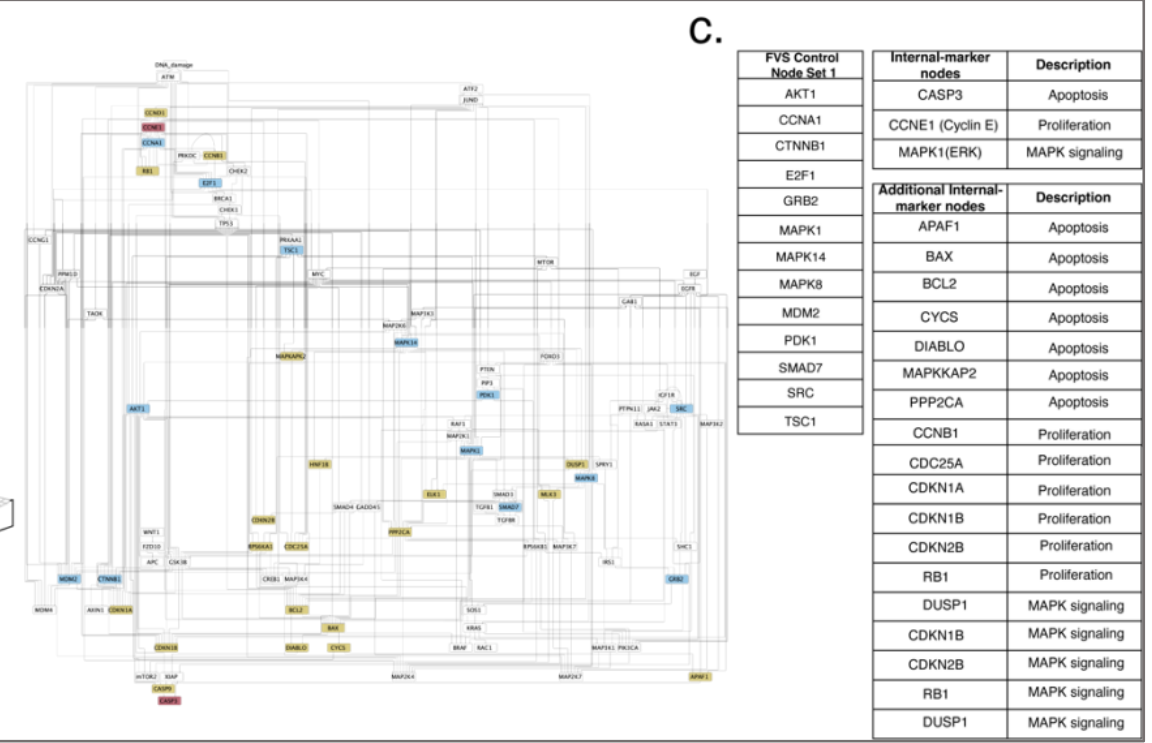

Fig. 7 Overcoming adaptive resistance in the Colorectal Cancer model. a In this study, we use NETISCE to identify perturbations on FVS control nodes that shift BRAFi-treated HT29 cells from a state of resistance to MAPK inhibitor (MAPKi) therapy and towards a MAPKi therapy sensitivity. We initialize the system with gene expression data from untreated HT29 cells $\left(x_{0}\right)$, and simulate BRAFi together with perturbations on FVS control nodes to shift the system to a state (yellow arrow leading to $x^{\prime}{ }_{0}$ ) that leads to sensitivity to MAPKi therapy (purple circle). b Colorectal cancer signaling network introduced in Park et al..$^{56}$. Blue nodes are control nodes, magenta nodes are internalmarker nodes, and gold nodes are additional internal-marker nodes used for further filtering of perturbations of FVS control nodes. c Key nodes for the cell fate reprogramming. Presented here is one of the 68 FVS control node sets within the network, called Set 1 . There are three internal-marker nodes used by Park et al. to identify targets to reprogram the system towards the MAPKi therapy-sensitive state. The three nodes are used to measure the phenotypes of apoptosis, proliferation, and MAPK signaling activity within the perturbed system. We identified an additional 17 internal-marker nodes to filter perturbations on FVS control nodes. The phenotypes associated with the internal-marker nodes are denoted in the second column.

194 The normalized gene expression data from an untreated HT29 sample was used for the initial activities for all SFA simulations, as data for HT29 cells with BRAFi and BRAFi+EGFRi

treatment is unavailable. In HT29 cells, PIK3CA and BRAF have gain-of-function mutations,

197 while APC, SMAD4, and TP53 have loss-of-function mutations. Therefore, states of nodes with

198 gain-of-function/loss-of-function mutations were overridden to the appropriate overexpression or

199 knockout state using our modified SFA equation for perturbations (see Methods). Next, we

200 simulated the treatment of an untreated HT29 cell with BRAFi (HT29_BRAFi) or BRAFi+EGFRi

201 (HT29_BRAFi+EGFRi) to obtain attractors related to the MAPKi therapy-resistant state and 
data of the untreated HT29 sample as initial state values and included the appropriate mutational overrides. To simulate BRAFi and EGFRi, the state of these nodes was overridden to a knockout state using the modified SFA equation for perturbations. The optimal k-means clustering of the attractors obtained from the untreated HT29, HT29_BRAFi, HT29_BRAFi+EGFRi treated initial conditions, and the attractors from 100,000 randomly generated initial states, was k=3; as desired, the untreated-state, MAPKi therapy-resistant state, 209 and MAPKi therapy-sensitive state associated attractors were found in separate clusters.

210 NETISCE identified 68 FVSes in the CRC static signaling network; the union of all FVSes 211 contained 25 nodes, and each FVS had a combination of 14 out of the 25 nodes. All FVSes 212 contained SRC. In addition, each FVS included TP53, a loss-of-function mutant gene in HT29 213 cells whose state was already overridden to a knockout state in our simulations; therefore,

214 additional perturbations to TP53 were not performed, reducing the number of FVS control nodes 215 that could be perturbed to 13 (Supplementary Data 1).

216 We present the results of using one FVS, referred to as Set 1 (Figure 7c), to identify

217 combinations of perturbations that shift the system from the MAPKi therapy-resistant phenotype

218 to the MAPKi therapy-sensitive phenotype. We simulated 1,594,323 combinations of

219 perturbations to the 13 FVS control nodes in Set 1 to identify their corresponding attractors.

220 First, 232,114 of the $1,594,323$ attractors generated from the perturbations on FVS control

221 nodes were classified to the MAPKi therapy-sensitivity associated cluster by at least two out of

222 three machine learning classification algorithms. Then, the 232,114 perturbations that produced

223 the attractors that passed criterion 1 were filtered by the three internal-marker nodes that were

224 used as output readout nodes in Park et al. (Figure 7c): CASP3 (apoptosis marker), Cyclin E

225 (proliferation marker), and MAPK1/ERK (MAPK signaling activity marker). The internal-marker

226 nodes in the attractors produced by 52,703 of the 232,114 perturbations on FVS control nodes

227 had steady-state expression values in the range of gene expression values of the MAPKi 


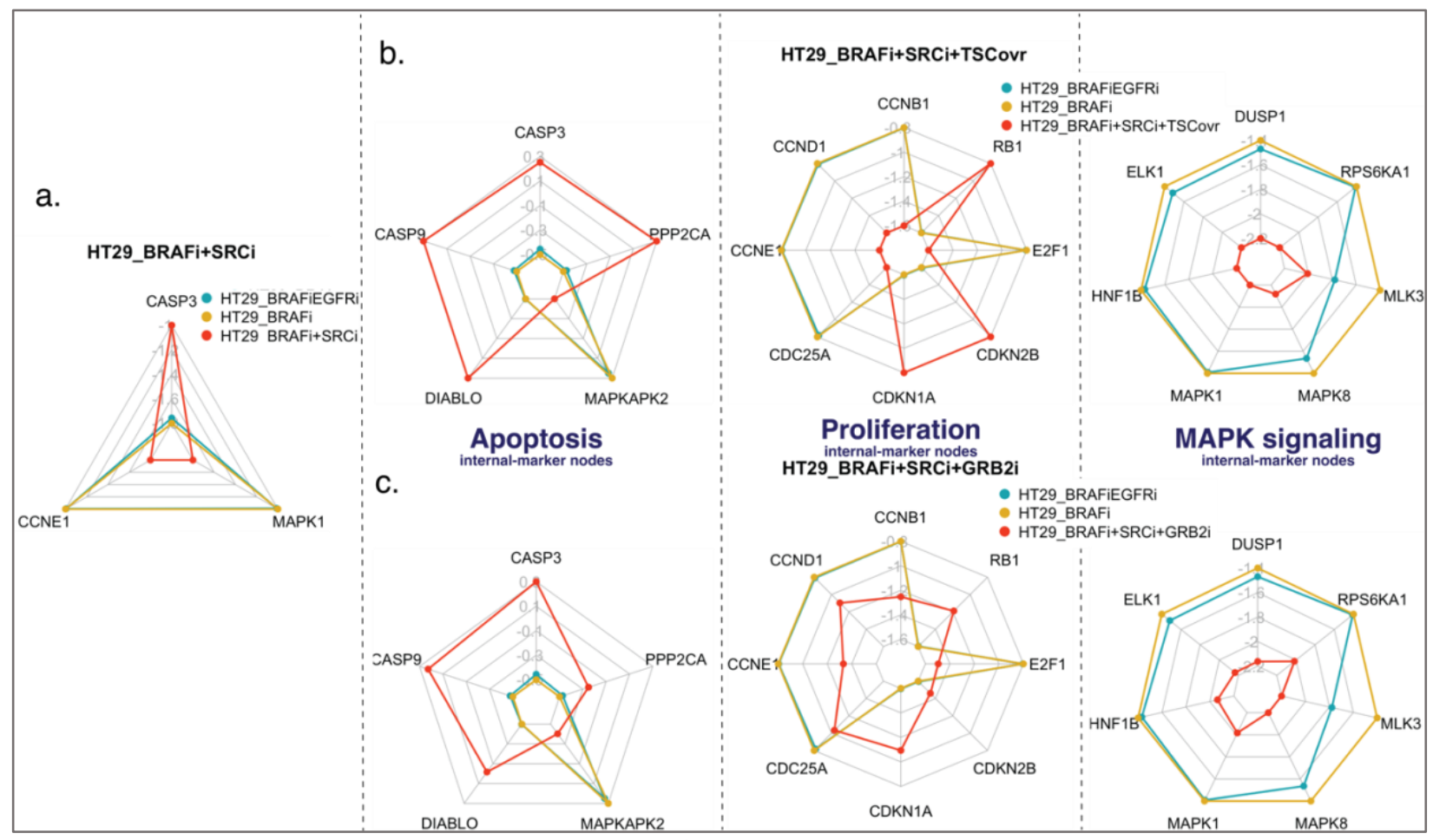

Fig. 8 Results of simulations of perturbations on FVS control nodes for overcoming adaptive resistance in colorectal cancer. Radar plots for perturbations on FVS control nodes in HT29 cells in the colorectal cancer signaling network. The title of each plot indicates the perturbation on the FVS control nodes with BRAFi. Each axis on the radar plot displays the steady-state expression value for an internal-marker node. The blue polygons are the steady-state expression values of the internal-marker nodes in the HT29_BRAFi+EGFRi (MAPKi therapy-sensitive) associated attractor. The yellow polygons are the average steady-state expression values of the internal-marker nodes in the HT29_BRAFi (MAPKi therapy-resistant) associated attractor. The orange polygons are the steady-state expression values of the internal-marker nodes in the attractor produced by the specified perturbation on FVS control nodes with BRAFi. A perturbation is considered successful if $90 \%$ of the internal-marker nodes' steady-state expression values are within the gene expression range of the HT29_BRAFi+EGFRi-associated attractor. a Radar plot of the three internal-marker nodes used by Park et al. for the simulated BRAFi+SRCi perturbation. The radar plot shows that the perturbation of BRAFi+SRCi increases apoptosis and decreases proliferation and MAPK activity based on the internal-marker nodes. $\mathbf{b}$ Radar plots for the two smallest sets of perturbations on FVS control nodes ( $\mathrm{SRCi}+\mathrm{TSC} 1$ overexpression, and SRCi+GRB2i) with 20 internal-marker nodes, separated by the apoptosis, MAPK signaling, and proliferation phenotypes. The plots show that for all internal-marker nodes, the internal-marker node values are within the ranges of the MAPK sensitivity associated phenotype.

230 We considered an additional 17 internal-marker nodes related to apoptosis, proliferation, and

231 MAPK signaling (Figure 7c). Of the 52,703 perturbations on FVS control nodes, 1,266 met the

232 filtering threshold of $90 \%$ for the apoptosis, proliferation, and MAPK internal-marker node

233 steady-state values. SCRi was present in all combinations of control nodes that passed the two 
235 The smallest sets of perturbations were two pairs of two control nodes. The first reprogramming

236 pair consisted of SRCi and TSC1 overexpression (SRCi+TSC1ovr) (Figure 8b), while the

237 second reprogramming pair was comprised of SRCi and GRB2i (SRCi+GRB2i) (Figure 8c).

238 TSC1 overexpression has been studied in the context of adaptive resistance to MAPKi. TSC1

239 promotes cell death by inhibiting mTOR activity, and mTOR inhibition in combination with BRAFi

240 has been shown to overcome adaptive resistance in BRAF-mutant melanoma ${ }^{58}$. However, a

241 complex of TSC1 and TSC2 can be inactivated by ERK phosphorylation, leading to increased

242 mTOR signaling ${ }^{59}$. In the context of CRC, GRB2 is an important protein for transmitting

243 oncogenic signaling and promoting tumorigenesis and metastasis ${ }^{60}$. Interestingly, the protein

244 Gab2, a binding partner of GRB2, was found to be directly upregulated by BRAFi in BRAF

245 mutated cancers ${ }^{61}$. Impairing the interaction between GRB2 and Gab2 sensitized cells to BRAFi

246 therapy and prevented additional oncogenic signaling and metastasis in HT29 cells ${ }^{61,62}$.

\section{DISCUSSION}

248 With the rise in availability of multi-omics datasets and tools for constructing gene regulatory

249 and intracellular signaling networks from these data, there is a growing need for cell

250 reprogramming methods that are data-driven and amenable for larger-scale biological networks

251 where parameters to model all the system components may not be available or be difficult to

252 estimate. We have developed NETISCE, a tool that identifies cell fate reprogramming targets

253 using the FVS control, attractor landscape estimation, and machine learning methods. By

254 reproducing experimental and mathematical model results, we show that NETISCE can identify

255 cell fate reprogramming targets and their perturbations using the system's static network, gene

256 expression data from the undesired cell phenotype, and a set of nodes used as internal-markers

257 for the desired and undesired phenotypes.

258 NETISCE offers a unique approach to identifying cell fate reprogramming targets through its 259 application of control theory and a dynamical systems-based framework. First, by employing the structure-based FVS control, we consider feedback loops that commonly regulate biological 
261 functions essential for identifying reprogramming targets. Since our target search focuses on

262 FVS control nodes, we guarantee that the identified nodes are sufficient for cell fate

263 reprogramming. Applying the FVS control to the problem of identifying cell fate reprogramming

264 targets has distinct advantages. In comparison to network-based approaches, we do not need

265 to compare the structure of multiple networks to identify cell fate reprogramming targets; in

266 contrast to dynamical systems-based methods that do not apply control theory, full dynamical

267 information for all network components is not required, nor is there a need to screen all network

268 elements. Additionally, FVS control contrasts with other control theory approaches for identifying

269 targets like Data Guided Control, which may fail to capture cell reprogramming dynamics due to

270 its linear assumption of regulatory dynamics ${ }^{41}$. It is important to note that FVS control for

271 identifying reprogramming targets requires a high-fidelity static network. As Mochizuki and

272 colleagues observed ${ }^{5}$ and later, Kobayashi and colleagues showed experimentally ${ }^{51,52}$, if a cell

273 state cannot be reached by perturbations to the FVS control nodes, then the FVS was not

274 correctly identified, and network revision should be performed.

275 Secondly, the SFA algorithm and machine learning methods allow us to identify the specific

276 perturbations on FVS control nodes required for cell fate reprogramming by estimating system

277 dynamics and the attractor landscape. In our approach, by associating phenotypes to the

278 attractor landscape via k-means clustering and classifying the attractors produced by

279 perturbations on FVS control nodes via machine learning classification methods, we observe

280 when the system has shifted towards the attractor associated with the desired phenotype.

281 Lastly, we have modified the original SFA algorithm ${ }^{47}$ to apply FVS control-based perturbations.

282 Unlike the SFA control method introduced by Lee and $\mathrm{Cho}^{47}$, where edge modifications

283 (removals or additions) were implemented to perform perturbations, our method of perturbations

284 to a node's state maintains the FVS of the network. Additionally, we have implemented

285 permanent overrides on FVS control nodes rather than the original form of SFA perturbations

286 that are transiently applied by changing the initial states of nodes. Transient perturbations are 
not applicable in the context of FVS control. First, as defined by Fielder et al. and Mochizuki et

al., overrides to the states of the FVS control nodes guarantee that the system will arrive at any desired attractor ${ }^{5,6}$. Second, our implementation mimics the experimental perturbations in Kobayashi et al., where FVS genes were permanently overexpressed or knocked out in the ascidian embryo. While transient perturbations could be used to simulate some types of singledrug treatments, their use in the context of the FVS control may not produce the desired

293 reprogramming.

294 NETISCE reproduced the simulated and experimentally validated results in applications of cell 295 fate reprogramming. Importantly, we have shown that NETISCE can be used to personalize 296 simulations on a network when provided with expression (and, if available, mutational profiles) data for a specified sample. In the cell fate specification in ascidian embryos example, our SFA simulations had an overall success of $85 \%$ in reproducing the experimentally validated perturbations. The inability to induce the pan-neural tissue fate by Neurog overexpression may be explained by the biological process of pan-neural tissue specification. Otx - a gene downstream of Neurog involved in pan-neural cell fate specification - is inactive in the ascidian embryo until the 32-cell stage of development ${ }^{63}$. Since overexpression of Neurog was performed at the start of the simulation, we may not be accurately simulating the timing of its

304 pan-neural inducing effect. A future update to the SFA algorithm could perform asynchronous 305 stochastic simulations, allowing for time-delayed perturbations, and potentially producing 306 information regarding the specific timings of perturbations to be performed for successful 307 cellular reprogramming ${ }^{41,64}$.

308 Our FVS control-guided method reduces the number of simulations needed to be performed to 309 identify targets for cell fate reprogramming. In the reprogramming tasks for the pluripotent stem 310 cells and CRC problems, some of the perturbations to the FVS control nodes that NETISCE 311 identified as successful were a subset of the perturbations found in Boolean models, where 312 reprogramming targets were identified by simulating perturbations to every node in the system. 
313 In addition, NETISCE revealed combinatorial strategies for cell fate reprogramming in both

314 models. In the case of the pluripotent stem cell model, Nanog is essential to maintain

315 pluripotency and additional overexpression of Klf4 could make the reprogrammed cell

316 unreceptive to extracellular signaling that may signal to exit from pluripotency, preserving high

317 levels of Nanog activity ${ }^{55}$. In the model of adaptive resistance to MAPKi therapy in CRC, the

318 combination of $\mathrm{BRAFi}+\mathrm{SRCi}+\mathrm{TSC} 1$ ovr could further increase sensitivity to treatment and the

319 rate of apoptosis by MAPK and mTOR signaling inhibition ${ }^{59}$. Alternatively, BRAFi+SRCi+GRB2i

320 can increase sensitivity to MAPKi therapy and prevent the metastatic spread of CRC tumors ${ }^{61}$.

321 With the potential to identify hundreds of thousands of perturbations that satisfy NETISCE's

322 filtering criteria, a method for prioritizing perturbations on FVS control nodes is essential. A

323 simple method employed in NETISCE is generating a secondary set of internal-marker nodes to

324 filter perturbations. Depending on the system, it may also be beneficial to prioritize the smallest

325 combinations of perturbations on FVS control nodes to ease experiments or prevent off-target

326 effects. Another prioritization approach could score perturbations based on the strength of their

327 effect on the target phenotypes while minimizing side-effects, similar to the method

328 implemented by Park et al. in the CRC Boolean model simulations ${ }^{56}$. A node received a high

329 score if, when the probability of its activity in the results of the asynchronous stochastic

330 simulations indicated inhibition, it would prevent adaptive resistance via ERK reactivation,

331 promote the therapeutic side-effect of increased apoptosis, and prevent the adverse side-effect

332 of increased proliferation. This scoring could be modified for NETISCE. Majorly, NETISCE

333 would need to perform stochastic asynchronous simulations like the Boolean Model simulation

334 framework used by Park and colleagues. The scoring also needs to consider overexpression,

335 knockout, and combinations of perturbations on FVS control nodes. Next, the processing of

336 internal-marker nodes could be modified to consider nodes related to side-effects. Finally, an

337 algorithm for path-finding and determining the effect of a perturbation on FVS control nodes on

338 off-target nodes would need to be implemented. 
339 In the context of stem-cell reprogramming, where changes to the epigenetic profile are a significant factor in reprogramming efficiency ${ }^{65}$, implementing a scoring of combinations of

341 perturbations on FVS control nodes that considers epigenetic information could be highly

342 effective to rank reprogramming targets. This information, which is incorporated into the tool,

343 IRENE, for both GRN construction and scoring of potential transcription factor-reprogramming

344 targets, increased reprogramming protocol efficiencies in some cases by more than $900 \%{ }^{37}$.

345 Similar to the method of Park et al., this would also require implementing a stochastic simulation

346 framework and could only be considered if the epigenetic information was available for both the

347 undesired and desired states.

348 Additionally, if applicable, such as in a disease model, information like druggability or drug synergies could be incorporated into prioritizing combinations of perturbations on FVS control 350 nodes $^{66,67}$. For example, PHAROS $^{67}$, a meta-database of drug-target information, can be used

351 to assess the druggability of SRC, GRB2, and TSC1. Currently, six drugs are approved SRC

352 inhibitors. However, there are no approved drugs for GRB2 inhibition nor known drugs or

353 molecules that bind to TSC1 and promote its overexpression. Therefore, SRCi would be a likely

354 first candidate for preventing adaptive resistance in combination with BRAFi.

355 Our dynamical systems-based analysis using static biological networks and experimental data 356 to estimate the attractor landscape and perform combinations of perturbations to control nodes

357 provides a valuable tool for intracellular signaling analysis. Because NETISCE can be applied to 358 biological networks of a larger scale that are not fully parameterized, we envision it as a primary 359 tool for cell fate reprogramming studies. Experimentalists can use the results generated from

360 NETISCE to prioritize wet-lab perturbation experiments. At the same time, mathematical

361 modelers can focus model construction towards regions that appear to be more relevant to the

362 desired reprogramming task. Finally, our method produces useful and potentially novel

363 combinations of perturbations for cell fate reprogramming that could eventually be applied for

364 treatments in disease models to recover healthy cell phenotypes in biological systems. 


\section{METHODS}

\section{$366 \quad$ NETISCE Overview}

367 INPUT. There are three required inputs for NETISCE: (1) a static network representing a 368 biological system, (2) a set of normalized gene expression data from cells with an undesired 369 phenotype, and (3) a set of internal-marker nodes - user-defined nodes within the network that 370 can be used as a point of reference to verify that their gene expression levels match the expected 371 values in desired and undesired phenotypes. Normalized gene expression data for cells with the 372 desired phenotype can also be provided (see the Pluripotent Stem Cell example) but is not 373 required for all use cases, such as simulating adaptive resistance to treatment (see the Colorectal

374 Cancer example). Optionally, the input can include mutational data to further specify the rules for 375 the network's simulations.

376 Step 1. Estimation of the attractor landscape. The goal of the first step of NETISCE is to estimate 377 the region of the attractor landscape containing steady-states associated with the desired and 378 undesired phenotype (Figure 2b). The network is simulated using an adapted version of SFA ${ }^{46}$. 379 SFA estimates signal flow, the information conveyed by a series of reactions as represented in a 380 signaling network or GRN, based only on topological information in the network and an initial state 381 of the network nodes. The output of SFA is the logarithm of the steady-state value, which we refer 382 to as the steady-state expression value for each network node. The initial states of the network 383 nodes are based on the normalized gene expression levels. We simulate the system using SFA 384 for each provided experimental sample until reaching the attractor.

385 We generate randomly sampled initial states and apply SFA to compute a sufficiently large 386 number of attractor states ${ }^{34}$. All the computed attractors are then clustered via k-means clustering.

387 The elbow and silhouette metrics are calculated to determine an optimal $\mathrm{k}^{51,52}$. The clusters are 388 also evaluated using the internal-marker node values.

389 Step 2. Virtual screenings on FVS control nodes. In this step, NETISCE identifies FVS control nodes and simulates combinations of perturbations to their activity (Figure 2c). First, the FVS 
391 control nodes are identified via a simulated annealing algorithm to determine the FVS of the

392 network ${ }^{53}$. Then, virtual screenings of combinations of perturbations on the FVS control nodes

393 are performed using the SFA algorithm. In these simulations, the initial states of the network

394 nodes are set to the normalized expression values of the cells with an undesired phenotype. We

395 have modified the SFA pipeline to implement overrides to control nodes to simulate 396 overexpression, knockout or no change to a node's activity.

397 Step 3. Filtering sets of perturbations on the FVS control nodes. The final step of the pipeline aims

398 to identify the combinations of perturbations on the FVS control nodes that result in the desired

399 cell fate reprogramming (Figure 2c). We employ two filtering criteria to evaluate the combinations

400 of perturbations on FVS control nodes. The first criterion uses Random Forest, Support Vector

401 Machine, and Naïve Bayes machine learning classification algorithms to classify the attractors

402 generated by perturbations on FVS control nodes. In this classification step, using the previously

403 clustered attractors by the k-means analysis, the attractors generated by perturbations on FVS

404 control nodes are classified either in the cluster(s) associated with the undesired or desired

405 phenotype ${ }^{54-56}$. To pass this filtering criterion, an attractor generated from the perturbation on FVS

406 control nodes must be classified to the cluster associated with the desired phenotype by at least

4072 out of 3 classification algorithms. After passing the first criterion, perturbations on FVS control

408 nodes are evaluated by the second filtering criterion, which focuses on the steady-state

409 expression values of the internal-marker nodes. Perturbations on FVS control nodes where at

410 least $90 \%$ of the internal-marker node steady-state expression values are within the expression

411 ranges of the attractors associated with the desired phenotype pass this filtration step. These

412 criteria produce a final set of perturbations on FVS control nodes that are considered capable of

413 reprogramming from an undesired cell fate towards the desired cell fate. 


\section{Estimation of Steady-States using Signal Flow Analysis}

418 Signal Flow Analysis (SFA) is based on the Signal Propagation algorithm developed by Lee and

$419 \mathrm{Cho}^{47}$. The algorithm is a linear difference equation that computes the activity of a network node

420 at a given time in terms of the state of the network node at the previous time step, the effect

421 (activating or inhibiting), and weight of the influence of its $m$ incoming edges, and the initial state

422 of the node. Specifically, the logarithm $\left(\log _{2}\right)$ of the steady-state activity $x_{i}(t+1)$ of a node $i$ at

423 time $t+1$ is estimated by the initial state of the node and the activities of its regulators at time $\mathrm{t}$

424 using the following equation:

425

$$
x_{i}(t+1)=\alpha \sum_{j=1}^{m}\left(W_{i j} x_{j}(t)\right)+(1-\alpha) b_{i}
$$

426 where $x_{j}(t)$ is the logarithm of the activity of $j$, a node connected to $i$ by an incoming edge, at

427 time $t$. The $W_{i j}$ is the weight of the edge between node $j$ and node $i$, which represents how much

428 influence node $j$ exerts on node $i$ through the edge. $W_{i j}$ is defined as:

$$
W_{i j}=\frac{\operatorname{sign}(i j)}{\sqrt{\left(D_{\text {out }}\right)_{j}\left(D_{\text {in }}\right)_{i}}}
$$

430 where $\operatorname{sign}(i j)$ is the value of the edge between $i$ and $j$ ( 1 for activating edges, -1 for inhibiting 431 edges), $D_{\text {out }}$ is the out-degree of $j$, and $D_{\text {in }}$ is the in-degree of $i$. Finally, $b$ is the logarithm of the

432 initial state of node $i$ and $\alpha$ is a hyperparameter used to weigh the influence of the network 433 structure and initial node state on the Signal Flow. By default, in our pipeline, the hyperparameter

$434 \alpha$ is set to 0.9 to provide greater weight to the network topology rather than initial activity based on the parameter settings used in previous control studies using SFA ${ }^{68}$.

436 To identify an attractor of the system, the signal propagation equation is solved for all network 437 nodes until the difference between $x(t+1)$ and $x(t)$ is less than a tolerance threshold (by default, 438 this tolerance threshold is $10^{-6}$ ). The steady-state expression values of two attractors under 439 different simulation inputs can be compared by computing the difference between two logarithms 440 of the steady-state expression values produced from SFA, analogously to a logarithm of the fold- 
441 change $\left(\log _{2} \mathrm{FC}\right)$ in differential gene expression analysis ${ }^{47}$. Though the actual numerical value

442 cannot be used to measure the magnitude of the change in expression, positive difference values

443 indicate that the specified perturbation led to a shift in signal flow that increases the gene's activity

444 at the steady-state. In contrast, negative values predict a decrease in activity at the steady-state

445 due to the perturbation.

446 When estimating the attractor landscape, we begin by solving the signal propagation equation for

447 the system using the experimental data for each sample; the initial activity of network nodes is

448 set to the normalized expression values. In most cases, the number of experimental samples is

449 insufficient for landscape estimation. Therefore, NETISCE can generate randomly sampled initial

450 states $(100,000$ by default), whose initial state values are calculated from the ranges of the

451 normalized expression values for each of the supplied phenotypes in the experimental data.

452 Association of the Attractor Landscape Clusters to Experimental Phenotypes

453 We employ k-means clustering to partition the attractors estimated from the normalized

454 expression data and the randomly generated initial states. We confirm that the attractors

455 computed from the undesired and desired experimental samples are different. We use two metrics

456 to determine the optimal number of k clusters. The first is the elbow metric, which determines the

457 optimal $\mathrm{k}$ by finding the minimal intra-cluster variation ${ }^{69}$. The second is the silhouette metric, which

458 aims to identify the optimal $\mathrm{k}$ as the number of clusters with minimal intra-cluster variation and

459 maximal inter-cluster variations ${ }^{70}$. When the two metrics disagree on the optimal $k$, the smallest

460 of the potential optimal k-values is chosen where the attractors estimated from the undesired and

461 desired phenotype experimental samples do not appear within the same cluster(s).

462 Finally, we use the internal-marker nodes to confirm that the steady-state expression values of

463 these nodes agree with experimental data or literature. NETISCE checks that the steady-state

464 expression value of each internal-marker node in the attractors associated with the undesired

465 phenotype and desired phenotype matches the expected differential gene expression patterns.

466 In the scenario where only the experimental data for the undesired phenotype was provided for 
467 the initial states, NETISCE verifies that the cluster(s) containing the attractors generated from the

468 experimental data only have attractors where the internal-marker node steady-state expression

469 values are within the range of expression values of the undesired phenotype. If experimental data

470 for both the undesired and desired phenotype is supplied for initial states, then NETISCE confirms

471 that the attractors generated from the two phenotypes do not appear in the same cluster and that

472 their internal-marker node steady-state expression values are within the appropriate expression

473 value ranges. For example, consider a gene known to have a higher expression in cells in the

474 desired phenotype than that of the undesired phenotype. NETISCE will verify that the steady-

475 state expression value in the attractor associated with the desired phenotype is greater than the

476 value in the attractor associated with the undesired phenotype (i.e., that the difference between

477 the steady-state expression value for the internal-marker node in the attractor associated with the

478 desired phenotype and the undesired phenotype is positive). Also, when multiple samples are

479 given for each phenotype, NETISCE verifies that the steady-state expression values of the

480 internal-marker nodes in the attractors of the undesired phenotype do not overlap with the values

481 in the attractors of the desired phenotypes. If an overlap occurs, the internal-marker node is

482 unreliable for analysis to separate the attractors in the different phenotypes. Thus, if the values of

483 the internal-marker nodes do not match the literature or do not separate well between the

484 attractors of the undesired and desired phenotype clusters, the user may elect to revise network

485 structure, remove specific internal-marker nodes, or adjust simulation settings.

486 Identification of the minimal Feedback Vertex Set

487 Structure-based methods study the controllability of systems based solely on the structure of the 488 network ${ }^{5,71,72}$. In recent years, structure-based control methods for systems with non-linear 489 dynamics have been proposed. One such structure-based control method for non-linear dynamics 490 is the Feedback Vertex Set Control introduced by Mochizuki et al. ${ }^{5,6}$. Feedback Vertex Set Control 491 is a structure-based control method focused on the controllability of the system by restricting the 492 target states to attractors. Mochizuki et al. mathematically proved that for a network governed by 
non-linear dynamics like those of cell signaling, the control action of overriding the state variables of the feedback vertex set (FVS) into a targeted desired trajectory ensures that the system will asymptotically approach the desired trajectory. Consider a directed graph $G=(V, E)$ comprised of node set $V$ and edge set $E$. The node states of $G$ are described by the ODE

$$
\dot{x_{n}}=F_{n}\left(x_{n}, x_{I_{n}}\right), n=1,2, \ldots, N
$$

498 where for the dynamics $x$ of node $n \in V, I_{n}$ is the set of nodes that regulate node $n$, such that

499 self-regulatory loops $\left(n \in I_{n}\right)$ are only positive. Additionally, we assume $F_{n}$ satisfies decay

500 condition:

$$
\partial_{1}\left(F_{n}\left(x_{n}, x_{I_{n}}\right)<0\right.
$$

502 for all $n$ where $\partial_{1}$ is the partial derivative w.r.t. the first occurrence of $x_{n}$ and not $x_{I_{n}}$.

503 Definition 1.1: In $G$, a subset $I \subseteq V$ of nodes is Feedback Vertex Set (FVS) if and only if removal 504 of set $G \backslash I$ leaves a graph without directed cycles. An FVS is minimal if it does not contain a proper subset that is an FVS itself. For simplicity, in this paper, we will consider all the FVSes to be minimal.

Definition 1.2: In a dynamic system, a subset $J \subseteq V$ of nodes is a set of determining nodes if and only if two solutions satisfy $\lim _{x \rightarrow \infty} \widetilde{x_{J}}(t)-x_{J}(t) \rightarrow 0$ whenever $\lim _{x \rightarrow \infty} \widetilde{x_{n}}(t)-x_{n}(t) \rightarrow 0$ for all components $n \in J \subseteq V$.

In Fielder et al. and Mochizuki et al. these two definitions were proven to be equivalent for

511 dynamics in a network ${ }^{5,6}$. Therefore, observation of the long-term dynamics of the FVS is sufficient

512 to identify all possible attractors of an entire system. Controlling the dynamics of the FVS $513\left(x_{I}^{*}(t)-x_{I}(t) \rightarrow 0\right)$ is sufficient to drive the dynamics $x(t)$ of a whole system to converge on one 514 of any attractors $x^{*}(t)$.

515 The minimal Feedback Vertex Set problem is a well-known NP-hard problem. Many algorithms 516 have been developed to find the near-minimum FVS. Based on the implementation in Zañudo et 517 al. ${ }^{72}$, we use a simulated annealing local search approach, SA-FVSP, originally described in 
518 Galinier et al. ${ }^{53}$. SA-FVSP has been shown to outperform the greedy adaptive search procedure ${ }^{73}$.

519 A network may have multiple FVSes depending on the size and structure, but each FVS has the

520 same capabilities for controlling cell fates.

\section{Simulating Perturbations on FVS Control Nodes}

522 After identifying the FVS control nodes to be used for virtual screenings, combinations of

523 perturbations (overexpression/upregulation, knockouts/downregulation, or no change) to an FVS

524 node's activity are generated. NETISCE generates $3^{n}$ combinations of control nodes

525 perturbations, where $\mathrm{n}$ is the number of FVS control nodes.

526 The initial state of a node not contained in the FVS or an FVS node whose perturbation is "no

527 change" is set to the normalized expression value of the experimental sample(s) for the selected

528 undesired phenotype.

529 To simulate the perturbations on FVS control nodes, we modified the SFA algorithm to override

530 the activity of perturbed control nodes. Specifically, the values of the perturbed FVS control nodes

531 are fixed and unaffected by the incoming signal flow. The fixed state $p$ of an upregulated

532 (downregulated) FVS control nodes $i$ is defined as:

$$
\begin{gathered}
p_{i}^{\text {upregulated }}=\max \left(\operatorname{normexp}_{i}\right)+2.5\left(\max \left(\operatorname{normex}_{i}\right)\right) \\
p_{i}^{\text {downregulated }}=\min \left(\text { normexp }_{i}\right)-2.5\left(\min \left(\operatorname{normex}_{i}\right)\right)
\end{gathered}
$$

536 Where $\max \left(\right.$ normexp $\left._{i}\right)$ and $\min \left(\right.$ normexp $\left._{i}\right)$ are the maximum and minimum normalized 537 expression value of $i$ across the experimental samples of the undesired phenotype, respectively.

538 These equations are also used when gain-of or loss-of-function information from mutational data 539 is supplied to NETISCE. For example, the value of a node that represents a gene with a gain-of-

540 function mutation is fixed to the corresponding $p_{i}^{\text {upregulated }}$ based on the normalized gene 541 expression data. 


\section{$543 \quad$ Classification of Perturbations on FVS Control Nodes}

544 To systematically identify which perturbations on the FVS control nodes shifted the system away

545 from the attractor associated with the undesired phenotype and towards the desired phenotype,

546 we filter the resultant attractors with two criteria. Criterion 1 considers a combination of

547 perturbations on FVS control nodes successful if the perturbation's corresponding attractor is

548 classified to the cluster associated with the desired phenotype by at least two of three machine

549 learning classification algorithms. The three classification algorithms considered in NETISCE are

550 Naïve Bayes, Support Vector Machines, and Random Forest classifiers. Naïve Bayes and

551 Support Vector Machines are well suited for high dimensional datasets ${ }^{74,75}$, while Random Forest

552 Classifiers improve predictive accuracy and reduce over-fitting ${ }^{76}$. Criterion 2 focuses on the

553 steady-state expression values of the internal-marker nodes. In this second criterion, the attractor

554 obtained after simulating the system under the studied combination of perturbations on FVS

555 control nodes must have at least $90 \%$ of the steady-state expression values of its internal-marker

556 nodes within the expected gene expression value ranges of the desired attractors. This ensures

557 that beyond the machine learning classification based on the entire attractor, the known biological

558 internal-marker nodes have the expected values of the desired phenotype. By default, NETISCE

559 is set to strict filtering criteria, where the steady-state expression values of the attractor produced

560 by the control node perturbation must be within the range of the desired phenotype expression

561 values. For example, consider an internal-marker node whose steady-state expression values in

562 the attractors associated with the desired phenotype is 2.0. The steady-state expression values

563 in the attractors associated with the undesired phenotype are 1.0. For a perturbation on the FVS

564 control nodes to pass the filtering criterion, the steady-state expression value of the internal-

565 marker node must be greater than 2.0. Alternatively, the user can select a more relaxed filtering

566 threshold. In this case, for the example described above, a perturbation on the FVS control nodes

567 would pass the filtering criterion if the steady-state expression value of the internal-marker node

568 is greater than 1.0. All the perturbations to the FVS control nodes that pass both filtering criteria 
569 are considered to successfully shift the initial state to an attractor associated with the desired

570 phenotype. If NETISCE is run with replicates for the undesired phenotype, then a perturbation on

571 FVS nodes must pass the first filtering criteria on all replicates. All replicates are individually

572 analyzed in the second filtering criterion, and NETISCE produces a separate list of perturbations

573 that pass the criterion for each replicate. In our pluripotent stem cell example that contained three

574 replicates, the perturbations on FVS control nodes that passed both criteria in the replicates were

575 identical. However, users familiar with their data may be interested in perturbations that only work

576 for a subset of replicates.

577 Data for Developmental, Stem Cell, and Cancer Biology Validations

578 Cell fate specification in the ascidian embryo.

579 The network structure was obtained from Kobayashi et al. ${ }^{55}$. Since the focus of this example was

580 to reproduce the experimental results of embryonic cell fate specification using Feedback Vertex

581 Set Control and SFA, we performed computations separate from the NETISCE pipeline but using

582 the essential scripts (see GitHub repository and tutorial). Without available normalized gene

583 expression data for the unperturbed embryo, we performed in silico simulations to reproduce the

584 cell fate specification results with SFA. The attractor for an unperturbed embryo was estimated

585 by setting the initial activities of two genes necessary for normal embryonic development, Gata.a

586 and Zic-r.a, to 1 , representing an activated state ${ }^{63}$. All other nodes were initialized to 0 ,

587 representing an initial inactive activity. The attractor estimation function was employed to simulate

588 the seven perturbations to the FVS control nodes that induced the seven tissue fates

589 experimentally: (Foxa.A, Foxd, Erk Signaling, Neurog, Tbx6-r.b, and Zic-r.b.). Specifically, in

590 these simulations, Gata.a and Zic-r.a had initial activities set to 1 and all other nodes set to 0.

591 Then, the values of the FVS control nodes were overridden using the $p_{i}^{\text {upregulated }}$ or

$592 p_{i}^{\text {downregulated }}$ equations. Since there was no gene expression data, for all FVS control genes the

$593 \quad p_{i}^{\text {upregulated }}=2.5$ and $p_{i}^{\text {downregulated }}=-2.5$ The internal-marker node steady-state expression 
594 values for the unperturbed and perturbations on FVS node simulation results can be found in

595 Supplementary Table 1. A perturbation was considered successful in replicating the experimental

596 results if the difference of the steady-state expression values between the specified internal-

597 marker node for the relevant tissue in attractor generated from a perturbation on FVS control

598 nodes and the attractor generated from the unperturbed state was positive (Supplementary Table

599 2). These values were additionally graphed using radar plots to visualize the respective

600 upregulations for each perturbation (Figure 4).

601 Induced pluripotent stem cell reprogramming from primed to naïve pluripotency.

602 The intracellular signaling network for induced pluripotent stem cell signaling was obtained from

603 Yachie-Kinoshita et al. ${ }^{54}$. The normalized expression data for EpiSCs and ESCs were 604 downloaded from the Gene Expression Omnibus (GSE88928) ${ }^{77}$. There were three replicates for 605 each experimental sample. Each replicate was used separately as initial state values to simulate 606 the network, compute their associated steady-states, and perform perturbations on the FVS 607 control nodes. Initially, we selected as internal-marker nodes the four output nodes used in the 608 Boolean Model of Yachie-Kinoshita et al.: Oct4, Sox2, Nanog, and EpiTFs. Although these three 609 nodes were also FVS control nodes, they were used as internal-marker nodes to be consistent 610 with the output nodes in the Boolean simulations. To further filter our perturbations, we selected 611 additional internal-marker nodes from gene expression data provided by Yachie-Kinoshita et al. 61254 . Based on the gene expression data for the network nodes, there were six genes whose values 613 differed significantly between the ESC and EpiSC states. These included Lefty1, Pitx2, and Esrrb.

614 The three other genes, Tbx3, Gata6, and Klf4, were not included as internal-marker nodes as 615 they were FVS control nodes. Radar plots were used to visualize the perturbations of Klf4 616 upregulation, Nanog upregulation, and the combined Klf4+Nanog upregulation (Figure 6). 
620 Overcoming adaptive resistance to MAPK inhibitory therapy in colorectal cancers.

621 The colorectal cancer (CRC) tumorigenesis signaling network and annotated HT29 mutational

622 profile for network nodes was provided by Park et al. ${ }^{56}$. The RNA-seq from untreated HT29 cells

623 was obtained from the Cancer Cell Line Encyclopedia (CCLE) $)^{78}$.

624 In this study of adaptive resistance to MAPKi therapy in CRC, the ultimate therapeutic goal is to

625 decrease proliferation and increase apoptosis in tumor cells. In a method adapted from Beal et

626 al. ${ }^{57}$, we verify that CRC tumors' proliferation and apoptosis signatures are preserved under the

627 SFA simulation of the generic CRC network for patient tumors (Supplementary Text 2).

628 The network was simulated using as initial conditions the normalized expression and mutational

629 profile of an untreated HT29 as there was no available gene expression data for treated HT29

630 cells. As annotated in Park et al. ${ }^{56}$, PIK3CA and BRAF have gain-of-function mutations, while

631 APC, SMAD4, and TP53 have loss-of-function mutations in HT29 cells. Therefore, the values of

632 these nodes were fixed to the appropriate $p_{i}^{\text {upregulated }}$ or $p_{i}^{\text {downregulated }}$ value. To simulate BRAFi

633 (HT29_BRAFi), $p_{i}^{\text {downregulated }}$ override was applied to the state of BRAF. To simulate

634 BRAFi+EGRFi (HT29_BRAFi+EGRFi), BRAF and EGFRi also had the appropriate overrides

635 applied using the $p_{i}^{\text {downregulated }}$ equation. We used the FVS finding algorithm to search for a

636 sufficiently large number of FVS in the CRC network, which identified 68 FVSes of size 14. TP53

637 was removed from the FVSes since it was already fixed to its $p_{i}^{\text {downregulated }}$ value due to the loss-

638 of-function mutation in HT29 cells (Supplementary Data 1). Based on Feedback Vertex Set control

639 theory ${ }^{5}$, all FVSes of a system can guide the system to any of its natural attractors; therefore, we

640 randomly selected the first FVS identified by the algorithm to perform perturbations on. For

641 simulating perturbations on FVS control nodes, the system was initialized with the same

642 parameters as the (HT29_BRAFi) simulation, with the additional perturbations to the FVS control

643 nodes. 
644 Control node perturbations were filtered first by the set of 3 internal-marker nodes used by Park

645 et al. ${ }^{56}$ : CASP3, a marker of apoptosis, CCNE1 (also known as Cyclin E), a proliferation marker, 646 and ERK (also known as MAPK1), a downstream molecule of the MAPK signaling pathway whose

647 activity after BRAFi treatment indicates adaptive resistance. Potential additional internal-marker

648 nodes were selected from downstream signaling elements of MAPK signaling, apoptosis-related,

649 and proliferation-related pathways ${ }^{56}$. These sets were filtered using the internal-marker node

650 checking step of NETISCE to ensure that their steady-state expression values in the attractors

651 associated with MAPK inhibitor therapy and MAPK inhibitor resistance were appropriate based

652 on literature evidence. This resulted in an additional 17 internal-marker nodes: four genes from

653 apoptosis-related pathways (CASP9, DIABLO, MAPKAPK2, PPP2CA), seven genes related to

654 proliferation (CCNB1, CCND1, CDC25A, CDKN1A, CDKN2B, E2F1, RB1), and six genes from

655 MAPK signaling pathways (DUSP1, ELK1, HNF1B, MAPK8, MLK3, RPS6KA1).

656 NETISCE Implementation

657 The main computational scripts of our pipeline are written in Python, utilizing the extensively 658 optimized machine learning algorithms of the Scikit Learn package ${ }^{79}$. Scripts for analyzing the 659 internal-marker node values are written in R. NETISCE is implemented as a Nextflow workflow ${ }^{80}$. 660 Nextflow is a state-of-the-art workflow manager tool that is language agnostic and designed for 661 parallel processing as a dataflow manager. Checkpoints are implemented for the user to 662 investigate any possible errors or make changes to run configuration, and the code can easily be 663 resumed without having to re-run all computations. We provide Nextflow pipelines for local 664 machine use and high-performance cluster implementations. We also provide NETISCE within a 665 Docker container to further enhance the reproducibility of NETISCE simulations ${ }^{81}$. In addition to 666 the command-line tool, our pipeline is available through the Galaxy Project, a cloud-based open667 source tool that requires little to no programming experience for biological analysis and 668 workflows ${ }^{82}$. 


\section{Data availability:}

671 The NETISCE's Nextflow pipeline version, the Docker image documentation, and data are

672 available at https://github.com/veraliconaresearchgroup/netisce. The installation, tutorials,

673 information for installing the Galaxy Project version of NETISCE, and walkthroughs for

674 reproducing the above results are found at https://veraliconaresearchgroup.github.io/Netisce/.

\section{Supplementary Files:}

676 Supplementary Information 1: supplementary-file.docx

677 Supplementary Data 1: supplementary-data1.xlsx

\section{Acknowledgements:}

679 We thank Pedro Mendes and Ion Moraru for their insightful discussions and feedback in the

680 methodology and validation of NETISCE. S.B. was supported by the UConn Health Research

681 Program; A.P. and M.S. worked on this project under the UConn High School Mentored

682 Research Program.

683 Author contributions:

684 P.V-L. and L.M. worked in the conceptualization and planning of this work. L.M, M.S., and A.P. 685 performed and analyzed the validation simulations. L.M, S.B., and M.S. participated in the 686 coding and implementation of NETISCE in Nextflow and Galaxy Project. L.M. and P.V-L. drafted 687 the manuscript. All authors read and approved the manuscript.

\section{Competing Interests:}

689 The authors do not report any competing interests.

\section{References:}


690 1. Liu, Y.-Y., Slotine, J.-J. \& Barabási, A.-L. Controllability of complex networks. Nature 473,

691

692

693

694

695

696

697

698

699

700

701

702

703

704

705

706

707

708

709

710

711

712

713

714

715

716

717

718

719

720

721

722

723

724

725

726

727

728

729

730

731

732

733

734

735

736

737

738

739

740
167-173 (2011).

2. Liu, Y.-Y., Slotine, J.-J. \& Barabási, A.-L. Observability of complex systems. Proc. Natl. Acad. Sci. U. S. A. 110, 2460-5 (2013).

3. Müller, F.-J. \& Schuppert, A. Few inputs can reprogram biological networks. Nat. 2011 4787369 478, E4-E4 (2011).

4. Cornelius, S. P., Kath, W. L. \& Motter, A. E. Realistic Control of Network Dynamics. doi:10.1038/ncomms2939.

5. Mochizuki, A., Fiedler, B., Kurosawa, G. \& Saito, D. Dynamics and control at feedback vertex sets. II: a faithful monitor to determine the diversity of molecular activities in regulatory networks. J. Theor. Biol. 335, 130-46 (2013).

6. Fiedler, B., Mochizuki, A., Kurosawa, G. \& Saito, D. Dynamics and Control at Feedback Vertex Sets. I: Informative and Determining Nodes in Regulatory Networks. J. Dyn. Differ. Equations 25, 563-604 (2013).

7. Cowan, N. J., Chastain, E. J., Vilhena, D. A., Freudenberg, J. S. \& Bergstrom, C. T. Nodal Dynamics, Not Degree Distributions, Determine the Structural Controllability of Complex Networks. PLoS One 7, e38398 (2012).

8. Takahashi, K. \& Yamanaka, S. Induction of Pluripotent Stem Cells from Mouse Embryonic and Adult Fibroblast Cultures by Defined Factors. Cell 126, 663-676 (2006).

9. Pera, M. F. \& Tam, P. P. L. Extrinsic regulation of pluripotent stem cells. Nat. 2010 4657299 465, 713-720 (2010).

10. Young, R. A. Control of the embryonic stem cell state. Cell 144, 940-954 (2011).

11. Gong, L. et al. Cancer cell reprogramming: a promising therapy converting malignancy to benignity. Cancer Commun. (London, England) 39, (2019).

12. Xiong, S., Feng, Y. \& Cheng, L. Cellular Reprogramming as a Therapeutic Target in Cancer. Trends Cell Biol. 29, 623-634 (2019).

13. Zimmermannova, O., Caiado, I., Ferreira, A. G. \& Pereira, C. F. Cell Fate Reprogramming in the Era of Cancer Immunotherapy. Front. Immunol. 12, (2021).

14. Gómez Tejeda Zañudo, J., Scaltriti, M. \& Albert, R. A network modeling approach to elucidate drug resistance mechanisms and predict combinatorial drug treatments in breast cancer. Cancer Converg. 1, 5 (2017).

15. Zañudo, J. G. T. et al. Towards control of cellular decision-making networks in the epithelial-to-mesenchymal transition. Phys. Biol. 16, 31002 (2019).

16. Liu, H. et al. DrugCombDB: a comprehensive database of drug combinations toward the discovery of combinatorial therapy. Nucleic Acids Res. 48, 871-881 (2019).

17. Mandai, M. et al. Autologous Induced Stem-Cell-Derived Retinal Cells for Macular Degeneration. N. Engl. J. Med. 376, 1038-1046 (2017).

18. Bosch, A. et al. PI3K inhibition results in enhanced estrogen receptor function and dependence in hormone receptor-positive breast cancer. Sci. Transl. Med. 7, 283ra51 (2015).

19. Koh, H. W. L. et al. iOmicsPASS: network-based integration of multiomics data for predictive subnetwork discovery. npj Syst. Biol. Appl. 201951 5, 1-10 (2019).

20. Zhang, M. et al. GeNeCK: a web server for gene network construction and visualization. BMC Bioinforma. 2019201 20, 1-7 (2019).

21. Shu, L. et al. Mergeomics: multidimensional data integration to identify pathogenic perturbations to biological systems. BMC Genomics 2016 171 17, 1-16 (2016).

22. Zhou, G. \& Xia, J. OmicsNet: a web-based tool for creation and visual analysis of biological networks in 3D space. Nucleic Acids Res. 46, W514-W522 (2018).

23. Cahan, P. et al. CellNet: Network Biology Applied to Stem Cell Engineering. Cell 158, 903-915 (2014).

24. Xu, Q. et al. ANANSE: an enhancer network-based computational approach for predicting 
key transcription factors in cell fate determination. Nucleic Acids Res. 49, 7966-7985 (2021).

25. Jia, D., Kumar Jolly, M., Kulkarni, P. \& Levine, H. cancers Phenotypic Plasticity and Cell Fate Decisions in Cancer: Insights from Dynamical Systems Theory. doi:10.3390/cancers9070070.

26. Greene, J. M., Gevertz, J. L. \& Sontag, E. D. Mathematical Approach to Differentiate Spontaneous and Induced Evolution to Drug Resistance During Cancer Treatment. JCO Clin. Cancer Informatics 1-20 (2019) doi:10.1200/cci.18.00087.

27. Tyson, J. J., Chen, K. C. \& Novak, B. Sniffers, buzzers, toggles and blinkers: dynamics of regulatory and signaling pathways in the cell. Curr. Opin. Cell Biol. 15, 221-31 (2003).

28. Kauffman, S. A. Metabolic stability and epigenesis in randomly constructed genetic nets. J. Theor. Biol. 22, 437-67 (1969).

29. Ferrell, J. E. Bistability, bifurcations, and Waddington's epigenetic landscape. Curr. Biol. 22, R458-66 (2012).

30. Choi, S. R., Hwang, C. Y., Lee, J. \& Cho, K.-H. Network Analysis Identifies Regulators of Basal-Like Breast Cancer Reprogramming and Endocrine Therapy Vulnerability. (2021) doi:10.1158/0008-5472.CAN-21-0621.

31. Huang, S., Ernberg, I. \& Kauffman, S. Cancer attractors: a systems view of tumors from a gene network dynamics and developmental perspective. Semin. Cell Dev. Biol. 20, 86976 (2009).

32. Cho, S. H., Park, S. M., Lee, H. Y. H. S., Lee, H. Y. H. S. \& Cho, K. H. Attractor landscape analysis of colorectal tumorigenesis and its reversion. BMC Syst. Biol. 10, 96 (2016).

33. Kim, Y., Choi, S., Shin, D. \& Cho, K.-H. Quantitative evaluation and reversion analysis of the attractor landscapes of an intracellular regulatory network for colorectal cancer. BMC Syst. Biol. 11, 45 (2017).

34. Cho, K.-H., Joo, J. II, Shin, D., Kim, D. \& Park, S.-M. The reverse control of irreversible biological processes. Wiley Interdiscip. Rev. Syst. Biol. Med. 8, 366-377 (2016).

35. Zh, Xu, J., Brusch, L. \& Huang, S. Predicting Pancreas Cell Fate Decisions and Reprogramming with a Hierarchical Multi-Attractor Model. PLoS One 6, e14752 (2011).

36. Folguera-Blasco, N., Cuyàs, E., Menéndez, J. A. \& Alarcón, T. Epigenetic regulation of cell fate reprogramming in aging and disease: A predictive computational model. PLOS Comput. Biol. 14, e1006052 (2018).

37. Jung, S., Appleton, E., Ali, M., Church, G. M. \& del Sol, A. A computer-guided design tool to increase the efficiency of cellular conversions. Nat. Commun. 12, (2021).

38. Rackham, O. et al. Challenges for Computational Stem Cell Biology: A Discussion for the Field. Stem cell reports 16, 3-9 (2021).

39. Melton, C., Judson, R. L. \& Blelloch, R. Opposing microRNA families regulate selfrenewal in mouse embryonic stem cells. Nat. 20104637281 463, 621-626 (2010).

40. Felsher, D. W. Reversibility of oncogene-induced cancer. Curr. Opin. Genet. Dev. 14, 3742 (2004).

41. Ronquist, S. et al. Algorithm for cellular reprogramming. Proc. Natl. Acad. Sci. 114, 11832-11837 (2017).

42. Jiang, J. \& Lai, Y. C. Irrelevance of linear controllability to nonlinear dynamical networks. Nat. Commun. $201910110,1-10$ (2019).

43. Zañudo, J. G. T. \& Albert, R. Cell fate reprogramming by control of intracellular network dynamics. PLoS Comput. Biol. 11, e1004193 (2015).

44. Ferrell, J. E. Self-perpetuating states in signal transduction: positive feedback, doublenegative feedback and bistability. Curr. Opin. Cell Biol. 14, 140-148 (2002).

45. Pomerening, J. R. Uncovering mechanisms of bistability in biological systems. Curr. Opin. Biotechnol. 19, 381-388 (2008). 
792

793

794

795

796

797

798

799

800

801

802

803

804

805

806

807

808

809

810

811

812

813

814

815

816

817

818

819

820

821

822

823

824

825

826

827

828

829

830

831

832

833

834

835

836

837

838

839

840

841

842

46. Qi, K. et al. [Expression and significance of IKBKB in pulmonary adenocarcinoma A549 cells and its cisplatin-resistant variant A549/DDP]. Zhongguo Fei Ai Za Zhi 17, 363-8 (2014).

47. Lee, D. \& Cho, K.-H. Topological estimation of signal flow in complex signaling networks. Sci. Rep. 8, 5262 (2018).

48. Arakelyan, A., Nersisyan, L., Johnson, G., Riel-Mehan, M. \& Pico, A. PSFC: A pathway signal flow calculator app for cytoscape. F1000Research 4, 480 (2017).

49. Lee, H.-Y. et al. Identifying molecular targets for reverse aging using integrated network analysis of transcriptomic and epigenomic changes during aging. Sci. Reports 2021111 11, 1-13 (2021).

50. Marazzi, L., Gainer-Dewar, A. \& Vera-Licona, P. OCSANA+: optimal control and simulation of signaling networks from network analysis. Bioinformatics (2020) doi:10.1093/bioinformatics/btaa625.

51. Kobayashi, K., Maeda, K., Tokuoka, M., Mochizuki, A. \& Satou, Y. Controlling Cell Fate Specification System by Key Genes Determined from Network Structure. iScience 4, 281-293 (2018).

52. Kobayashi, K., Maeda, K., Tokuoka, M., Mochizuki, A. \& Satou, Y. Using linkage logic theory to control dynamics of a gene regulatory network of a chordate embryo. Sci. Rep. 11, 4001 (2021).

53. Galinier, P., Lemamou, E. \& Bouzidi, M. W. Applying local search to the feedback vertex set problem. J. Heuristics 19, 797-818 (2013).

54. Yachie-Kinoshita, A. et al. Modeling signaling-dependent pluripotency with Boolean logic to predict cell fate transitions. Mol. Syst. Biol. 14, 1-16 (2018).

55. Hara, M. R., Sachs, B. D., Caron, M. G. \& Lefkowitz, R. J. Pharmacological blockade of a $\beta_{2}$ AR- $\beta$-arrestin-1 signaling cascade prevents the accumulation of DNA damage in a behavioral stress model. Cell Cycle 12, 219-224 (2013).

56. Park, S.-M. M., Hwang, C. Y., Choi, J., Joung, C. Y. \& Cho, K.-H. H. Feedback analysis identifies a combination target for overcoming adaptive resistance to targeted cancer therapy. Oncogene 39, 3803-3820 (2020).

57. Béal, J., Montagud, A., Traynard, P., Barillot, E. \& Calzone, L. Personalization of Logical Models With Multi-Omics Data Allows Clinical Stratification of Patients. Front. Physiol. 9, 1965 (2019).

58. Paton, E. L., Turner, J. A. \& Schlaepfer, I. R. Overcoming Resistance to Therapies Targeting the MAPK Pathway in BRAF-Mutated Tumours. J. Oncol. 2020, (2020).

59. RB, C. et al. EGFR-mediated re-activation of MAPK signaling contributes to insensitivity of BRAF mutant colorectal cancers to RAF inhibition with vemurafenib. Cancer Discov. 2, 227-235 (2012).

60. Ding, C. B., Yu, W. N., Feng, J. H. \& Luo, J. M. Structure and function of Gab2 and its role in cancer (Review). Mol. Med. Rep. 12, 4007-4014 (2015).

61. Herr, R. et al. BRAF inhibition upregulates a variety of receptor tyrosine kinases and their downstream effector Gab2 in colorectal cancer cell lines. Oncogene 20183712 37, 1576-1593 (2018).

62. Bier, D. et al. Small-Molecule Stabilization of the 14-3-3/Gab2 Protein-Protein Interaction (PPI) Interface. ChemMedChem 11, 911-918 (2016).

63. Satou, Y. \& Imai, K. S. Gene regulatory systems that control gene expression in the Ciona embryo. Proc. Jpn. Acad. Ser. B. Phys. Biol. Sci. 91, 33-51 (2015).

64. Yang, G., Gómez Tejeda Zañudo, J. \& Albert, R. Target Control in Logical Models Using the Domain of Influence of Nodes. Front. Physiol. 9, 454 (2018).

65. Papp, B. \& Plath, K. Reprogramming to pluripotency: stepwise resetting of the epigenetic landscape. Cell Res. 21, 486-501 (2011).

66. Cotto, K. C. et al. DGldb 3.0: a redesign and expansion of the drug-gene interaction 
database. Nucleic Acids Res. 46, (2018).

67. Sheils, T. K. et al. TCRD and Pharos 2021: mining the human proteome for disease biology. Nucleic Acids Res. 49, D1334-D1346 (2021).

68. D, L. \& KH, C. Signal flow control of complex signaling networks. Sci. Rep. 9, (2019).

69. Satopää, V., Albrecht, J., Irwin, D. \& Raghavan, B. Finding a 'kneedle' in a haystack: Detecting knee points in system behavior. Proc. - Int. Conf. Distrib. Comput. Syst. 166171 (2011) doi:10.1109/ICDCSW.2011.20.

70. Rousseeuw, P. J. Silhouettes: A graphical aid to the interpretation and validation of cluster analysis. J. Comput. Appl. Math. 20, 53-65 (1987).

71. Ching-Tai Lin. Structural controllability. IEEE Trans. Automat. Contr. 19, 201-208 (1974).

72. Zañudo, J. G. T., Yang, G. \& Albert, R. Structure-based control of complex networks with nonlinear dynamics. Proc. Natl. Acad. Sci. U. S. A. 114, 7234-7239 (2017).

73. Pardalos, P. M., Qian, T. \& Resende, M. G. C. A Greedy Randomized Adaptive Search Procedure for the Feedback Vertex Set Problem. J. Comb. Optim. 2, 399-412 (1998).

858 75. Platt, J. C. Probabilistic Outputs for Support Vector Machines and Comparisons to Regularized Likelihood Methods. Adv. LARGE MARGIN Classif. 61 --74 (1999) doi:10.1.1.41.1639.

76. Breiman, L. Random Forests. Mach. Learn. 2001 451 45, 5-32 (2001).

77. D, K. et al. Endogenous WNT signals mediate BMP-induced and spontaneous differentiation of epiblast stem cells and human embryonic stem cells. Stem cell reports 4 , 114-128 (2015).

78. Barretina, J. et al. The Cancer Cell Line Encyclopedia enables predictive modelling of anticancer drug sensitivity. Nature 483, 603-7 (2012).

79. Pedregosa, F. et al. Scikit-learn: Machine Learning in Python. Journal of Machine Learning Research vol. 12 http://scikit-learn.sourceforge.net. (2011).

80. P, D. T. et al. Nextflow enables reproducible computational workflows. Nat. Biotechnol. 35, 316-319 (2017).

81. Merkel, D. Docker: lightweight Linux containers for consistent development and deployment: Linux Journal: Vol 2014, No 239. Linux J. 2014, 2 (2014).

82. V, J. et al. The Galaxy platform for accessible, reproducible and collaborative biomedical analyses: 2020 update. Nucleic Acids Res. 48, W395-W402 (2020). 